\title{
COERCIVE SPACE-TIME FINITE ELEMENT METHODS FOR INITIAL BOUNDARY VALUE PROBLEMS*
}

\author{
$\mathrm{OLAF}^{\mathrm{STEINBACH}}{ }^{\dagger}$ AND MARCO ZANK ${ }^{\ddagger}$
}

\begin{abstract}
We propose and analyse new space-time Galerkin-Bubnov-type finite element formulations of parabolic and hyperbolic second-order partial differential equations in finite time intervals. Using Hilbert-type transformations, this approach is based on elliptic reformulations of first- and second-order time derivatives, for which the Galerkin finite element discretisation results in positive definite and symmetric matrices. For the variational formulation of the heat and wave equations, we prove related stability conditions in appropriate norms, and we discuss the stability of related finite element discretisations. Numerical results are given which confirm the theoretical results.
\end{abstract}

Key words. space-time FEM, heat equation, wave equation

AMS subject classifications. 65M60

1. Introduction. While for the analysis of parabolic and hyperbolic partial differential equations a variety of approaches such as Fourier methods, semigroups, or Galerkin methods are available (see, for example, [22, 27, 28, 32, 44, 46]), standard approaches for the numerical solution are based on semi-discretisation, where the discretisation in space and time is split accordingly; see, e.g., [42] for parabolic partial differential equations and [7, 8] for hyperbolic problems. More recently, there exist space-time approaches as, for example, in [1, 11, 24, $30,31,34,37,41,43]$ for parabolic problems and [3, 5, 13, 17, 21, 29, 47] for hyperbolic equations.

In this work, we introduce a new Fourier-type method for the analysis of first- and secondorder ordinary differential equations, and we transfer this approach to the corresponding parabolic and hyperbolic partial differential equations. The aim of this work is to provide spacetime Galerkin-Bubnov-type variational formulations, where unique solvability follows from related coercivity estimates. This analysis may then serve not only as basis for the development and the numerical analysis of adaptive space-time finite element methods simultaneously in space and time and for the construction of time-parallel iterative solution strategies, but also for the analysis of related boundary integral equation methods for the heat and wave equation, respectively, and the coupling of finite and boundary element methods.

As a first model problem, we consider the Dirichlet boundary value problem for the heat equation,

$$
\begin{aligned}
\alpha \partial_{t} u(x, t)-\Delta_{x} u(x, t) & =f(x, t) & & \text { for }(x, t) \in Q:=\Omega \times(0, T), \\
u(x, t) & =0 & & \text { for }(x, t) \in \Sigma:=\Gamma \times(0, T), \\
u(x, 0) & =\quad 0 & & \text { for } x \in \Omega,
\end{aligned}
$$

where $\Omega \subset \mathbb{R}^{d}, d=1,2,3$, is a bounded domain with, for $d=2,3$, Lipschitz boundary $\Gamma=\partial \Omega, \alpha>0$ is a given heat capacity constant, and $f(x, t)$ is a given right-hand side. Note that in the spatially one-dimensional case $d=1$, we have $\Omega=(a, b)$ and $\Gamma=\{a, b\}$.

\footnotetext{
*Received December 21, 2018. Accepted February 7, 2020. Published online on April 1, 2020. Recommended by Simona Perotto.

$\dagger$ Institut für Angewandte Mathematik, TU Graz, Steyrergasse 30, 8010 Graz, Austria (o.steinbachetugraz.at).

${ }^{\ddagger}$ Fakultät für Mathematik, Universität Wien, Oskar-Morgenstern-Platz 1, 1090 Wien, Austria (marco.zank@univie.ac.at).
} 
A variational formulation of (1.1) is to find $u \in L^{2}\left(0, T ; H_{0}^{1}(\Omega)\right) \cap H_{0}^{1}\left(0, T ; H^{-1}(\Omega)\right)$ such that

$$
\begin{aligned}
\int_{0}^{T} \int_{\Omega} \alpha \partial_{t} u(x, t) v(x, t) d x d t+\int_{0}^{T} \int_{\Omega} \nabla_{x} u(x, t) & \cdot \nabla_{x} v(x, t) d x d t \\
& =\int_{0}^{T} \int_{\Omega} f(x, t) v(x, t) d x d t
\end{aligned}
$$

is satisfied for all $v \in L^{2}\left(0, T ; H_{0}^{1}(\Omega)\right)$, where we assume $f \in L^{2}\left(0, T ; H^{-1}(\Omega)\right)$. Note that we use the standard Bochner spaces, where $u \in H_{0}^{1},\left(0, T ; H^{-1}(\Omega)\right)$ satisfies $u(x, 0)=0$ for $x \in \Omega$. Related to the variational formulation (1.2), we introduce the bilinear form

$$
a(u, v):=\int_{0}^{T} \int_{\Omega}\left[\alpha \partial_{t} u(x, t) v(x, t)+\nabla_{x} u(x, t) \cdot \nabla_{x} v(x, t)\right] d x d t .
$$

Since (1.2) is a Galerkin-Petrov variational formulation, we need to establish an appropriate stability condition to ensure unique solvability; see also [14, 15, 34, 37, 43]. In particular,

$$
\|u\|_{L^{2}\left(0, T ; H_{0}^{1}(\Omega)\right) \cap H_{0,\left(0, T ; H^{-1}(\Omega)\right)}^{1}}:=\sqrt{\left\|\alpha \partial_{t} u\right\|_{L^{2}\left(0, T ; H^{-1}(\Omega)\right)}^{2}+\left\|\nabla_{x} u\right\|_{L^{2}(Q)}^{2}}
$$

defines a norm in $L^{2}\left(0, T ; H_{0}^{1}(\Omega)\right) \cap H_{0}^{1}\left(0, T ; H^{-1}(\Omega)\right)$, and we can prove the stability condition

$$
\frac{1}{\sqrt{2}}\|u\|_{L^{2}\left(0, T ; H_{0}^{1}(\Omega)\right) \cap H_{0,}^{1}\left(0, T ; H^{-1}(\Omega)\right)} \leq \sup _{0 \neq v \in L^{2}\left(0, T ; H_{0}^{1}(\Omega)\right)} \frac{a(u, v)}{\|v\|_{L^{2}\left(0, T ; H_{0}^{1}(\Omega)\right)}}
$$

for $u \in L^{2}\left(0, T ; H_{0}^{1}(\Omega)\right) \cap H_{0}^{1}\left(0, T ; H^{-1}(\Omega)\right)$. Since the bilinear form (1.3) is continuous, satisfying

$$
|a(u, v)| \leq \sqrt{2}\|u\|_{L^{2}\left(0, T ; H_{0}^{1}(\Omega)\right) \cap H_{0,\left(0, T ; H^{-1}(\Omega)\right)}^{1}}\|v\|_{L^{2}\left(0, T ; H_{0}^{1}(\Omega)\right)}
$$

for $u \in L^{2}\left(0, T ; H_{0}^{1}(\Omega)\right) \cap H_{0}^{1}\left(0, T ; H^{-1}(\Omega)\right)$ and $v \in L^{2}\left(0, T ; H_{0}^{1}(\Omega)\right)$, and surjective, this implies unique solvability of the variational problem (1.2); see, e.g., [6, 14]. The initial Dirichlet boundary value problem (1.1) therefore defines an isomorphism

$$
\mathcal{L}: L^{2}\left(0, T ; H_{0}^{1}(\Omega)\right) \cap H_{0,}^{1}\left(0, T ; H^{-1}(\Omega)\right) \rightarrow\left[L^{2}\left(0, T ; H_{0}^{1}(\Omega)\right)\right]^{\prime} .
$$

When considering the variational formulation (1.2) and performing integration by parts in time, this leads to the adjoint variational formulation to find $u \in L^{2}\left(0, T ; H_{0}^{1}(\Omega)\right)$ such that

$$
\begin{aligned}
-\int_{0}^{T} \int_{\Omega} u(x, t) \alpha \partial_{t} v(x, t) d x d t+\int_{0}^{T} \int_{\Omega} \nabla_{x} u(x, t) & \cdot \nabla_{x} v(x, t) d x d t \\
& =\int_{0}^{T} \int_{\Omega} f(x, t) v(x, t) d x d t
\end{aligned}
$$

is satisfied for all $v \in L^{2}\left(0, T ; H_{0}^{1}(\Omega)\right) \cap H_{, 0}^{1}\left(0, T ; H^{-1}(\Omega)\right)$, where the test space includes the final time condition $v(x, T)=0$ for $x \in \Omega$ and where we assume $f \in\left[L^{2}\left(0, T ; H_{0}^{1}(\Omega)\right) \cap\right.$ $\left.H_{, 0}^{1}\left(0, T ; H^{-1}(\Omega)\right)\right]^{\prime}$. As for the primal variational formulation (1.2), we can establish unique solvability of the adjoint variational formulation (1.5), which then implies an isomorphism

$$
\mathcal{L}: L^{2}\left(0, T ; H_{0}^{1}(\Omega)\right) \rightarrow\left[L^{2}\left(0, T ; H_{0}^{1}(\Omega)\right) \cap H_{, 0}^{1}\left(0, T ; H^{-1}(\Omega)\right)\right]^{\prime} .
$$


Both the primal variational formulation (1.2) and the adjoint variational formulation (1.5) are Galerkin-Petrov formulations, where the test space is different from the ansatz space, in particular with respect to time. This motivates to consider variational formulations for the initial boundary value problem (1.1), where ansatz and test spaces are of the same order also in time. Using the isomorphisms (1.4) and (1.6) and some interpolation arguments, one expects to consider test and ansatz spaces as subspaces of the anisotropic Sobolev space $H^{1,1 / 2}(Q)$, e.g., $[4,22,23,27,28]$. In the case of an infinite time interval, i.e., $T=\infty$, such an approach was considered analytically in the Ph.D. thesis of M. Fontes [16] (see also [25] for a related numerical analysis using wavelets) and the work of D. Devaud [10]. However, here we will consider only finite time intervals with $T<\infty$. In the case of time-periodic boundary value problems, a related approach is considered in [26].

Although the numerical analysis of space-time finite element methods for the variational formulation (1.2) is well-established (see, e.g., [1, 15, 30, 31, 34, 37, 43]), the analysis of boundary integral equations and related boundary element methods for the solution of the heat equation (1.1) relies on Galerkin-Bubnov variational formulations in anisotropic Sobolev trace spaces of $H^{1,1 / 2}(Q)$; see, e.g., [2, 4]. In particular, instead of a stability condition in the finite element analysis, an ellipticity estimate in the boundary element analysis is used. So, we are interested in a unified approach to analyse both finite and boundary element methods within one framework and allowing a numerical analysis also for the coupling of space-time finite and boundary element methods.

In addition to the initial boundary value problem (1.1) of the heat equation, we also consider the related model problem for the wave equation,

$$
\begin{aligned}
\frac{1}{c^{2}} \partial_{t t} u(x, t)-\Delta_{x} u(x, t) & =f(x, t) & & \text { for }(x, t) \in Q:=\Omega \times(0, T), \\
u(x, t) & =\quad 0 & & \text { for }(x, t) \in \Sigma:=\Gamma \times(0, T), \\
u(x, 0)=\partial_{t} u(x, 0) & =\quad 0 & & \text { for } x \in \Omega,
\end{aligned}
$$

where $c>0$ is a given wave speed constant. A standard approach for a space-time finite element method to solve (1.7) is to consider an equivalent system with first-order time derivatives; see, e.g., [3, 13, 29]. Alternatively, one may consider variational formulations of the wave equation in (1.7) using integration by parts also in time; see, e.g., [5, 17, 47]. Here, we will consider related variational formulations in suitable subspaces of $H^{1}(Q)$, and we will prove and discuss stability conditions in appropriate function spaces.

The rest of this paper is organised as follows: In Section 2 we consider simple first-order ordinary differential equations to motivate the choice of a transformation operator to derive an elliptic and symmetric bilinear form for the first-order time derivative. We discuss several properties of the Hilbert-type transformation operator, and we present some numerical results to illustrate the theoretical results. The results for the first-order ordinary differential equations are extended in Section 3 to the heat equation in several space dimensions. We prove that the heat partial differential operator with zero Dirichlet boundary and initial conditions defines an isomorphism in certain anisotropic Sobolev spaces, implying a stability condition as required in the numerical analysis of the proposed Galerkin scheme. We comment on the stability of the numerical scheme and present some numerical results. Second-order ordinary differential equations are considered in Section 4, where we introduce a different transformation operator, which is not semi-definite as in the case of first-order equations. Hence, we have to use different Sobolev norms to establish optimal stability estimates. As for the first-order equation, we provide a numerical analysis for the finite element discretisation, and we give some numerical results. Finally, in Section 5 we consider the space-time variational formulation for the wave equation, we discuss the discretisation scheme, and we provide some numerical results for illustration. 
2. First-order ordinary differential equations. As a first model problem, for $T>0$, we consider the simple initial value problem

$$
\partial_{t} u(t)=f(t) \quad \text { for } t \in(0, T), \quad u(0)=0,
$$

where we aim to derive and analyse a coercive variational formulation, which later will be used for the discretisation of time-dependent partial differential equations, which are of first-order in time.

2.1. Primal variational formulation. If we define the Sobolev space

$$
H_{0,}^{1}(0, T):=\left\{v \in H^{1}(0, T): v(0)=0\right\},
$$

then the primal variational formulation of (2.1) is to find $u \in H_{0,}^{1}(0, T)$ such that

$$
\int_{0}^{T} \partial_{t} u(t) v(t) d t=\int_{0}^{T} f(t) v(t) d t \quad \text { for all } v \in L^{2}(0, T) .
$$

Obviously, it is sufficient to assume that $f \in L^{2}(0, T)$ in this case. Recall that

$$
\|u\|_{H_{0,(0, T)}^{1}}^{2}:=\left\|\partial_{t} u\right\|_{L^{2}(0, T)}^{2}=\int_{0}^{T}\left[\partial_{t} u(t)\right]^{2} d t
$$

defines a norm in $H_{0,}^{1}(0, T)$. The bilinear form $a(\cdot, \cdot): H_{0}^{1}(0, T) \times L^{2}(0, T) \rightarrow \mathbb{R}$,

$$
a(u, v):=\int_{0}^{T} \partial_{t} u(t) v(t) d t
$$

is bounded, i.e.,

$$
|a(u, v)| \leq\left\|\partial_{t} u\right\|_{L^{2}(0, T)}\|v\|_{L^{2}(0, T)} \quad \text { for all } u \in H_{0,}^{1}(0, T), v \in L^{2}(0, T),
$$

and satisfies the stability condition

$$
\left\|\partial_{t} u\right\|_{L^{2}(0, T)} \leq \sup _{0 \neq v \in L^{2}(0, T)} \frac{a(u, v)}{\|v\|_{L^{2}(0, T)}} \quad \text { for all } u \in H_{0,}^{1}(0, T) .
$$

Moreover, it holds true that

$$
\|v\|_{L^{2}(0, T)} \leq \sup _{0 \neq u \in H_{0,(0, T)}^{1}} \frac{a(u, v)}{\left\|\partial_{t} u\right\|_{L^{2}(0, T)}} \quad \text { for all } v \in L^{2}(0, T) .
$$

As a consequence (see, e.g., [6, Satz 3.6] or [14, Corollary A.45]), we conclude unique solvability of the primal variational formulation (2.2), and the bilinear form (2.3) implies, by the Riesz representation theorem, a bounded and invertible operator

$$
B_{1}: H_{0,}^{1}(0, T) \rightarrow L^{2}(0, T)
$$

satisfying

$$
\|u\|_{H_{0,}^{1}(0, T)} \leq\left\|B_{1} u\right\|_{L^{2}(0, T)} \quad \text { for all } u \in H_{0,}^{1}(0, T) .
$$


2.2. Dual variational formulation. When using integration by parts, instead of the primal variational formulation (2.2), we may consider the dual variational formulation to find $u \in L^{2}(0, T)$ such that

$$
\int_{0}^{T} u(t) \partial_{t} v(t) d t=-\int_{0}^{T} f(t) v(t) d t \quad \text { for all } v \in H_{, 0}^{1}(0, T),
$$

where

$$
H_{, 0}^{1}(0, T):=\left\{v \in H^{1}(0, T): v(T)=0\right\}, \quad\|v\|_{H_{, 0}^{1}(0, T)}^{2}:=\int_{0}^{T}\left[\partial_{t} v(t)\right]^{2} d t .
$$

Here, it is sufficient to assume that $f \in\left[H_{, 0}^{1}(0, T)\right]^{\prime}$. As for the primal variational formulation, we conclude unique solvability of the dual variational formulation (2.4), which then implies a bounded and invertible operator

$$
B_{0}: L^{2}(0, T) \rightarrow\left[H_{, 0}^{1}(0, T)\right]^{\prime}
$$

satisfying

$$
\|u\|_{L^{2}(0, T)} \leq\left\|B_{0} u\right\|_{\left[H_{, 0}^{1}(0, T)\right]^{\prime}} \quad \text { for all } u \in L^{2}(0, T) .
$$

2.3. Interpolation of operators. Related to the initial value problem (2.1), we consider the operator $B_{1}: H_{0}^{1}(0, T) \rightarrow L^{2}(0, T)$ of the primal formulation (2.2) and the operator $B_{0}: L^{2}(0, T) \rightarrow\left[H_{, 0}^{1}(0, T)\right]^{\prime}$ of the dual formulation (2.4). Hence, using interpolation arguments for $s \in(0,1)$, we consider an operator

$$
B_{s}:\left[H_{0}^{1}(0, T), L^{2}(0, T)\right]_{s} \rightarrow\left[L^{2}(0, T),\left[H_{, 0}^{1}(0, T)\right]^{\prime}\right]_{s},
$$

and we may ask for a representation of $B_{s}$, in particular for $s=\frac{1}{2}$. Recall that the Sobolev space

$$
H_{0,}^{1 / 2}(0, T):=\left[H_{0,}^{1}(0, T), L^{2}(0, T)\right]_{1 / 2}
$$

is a dense subspace of $H^{1 / 2}(0, T)$ with the Hilbertian norm

$$
\|u\|_{H_{0}^{1 / 2}(0, T)}^{2}=\int_{0}^{T}[u(t)]^{2} d t+\int_{0}^{T} \int_{0}^{T} \frac{[u(s)-u(t)]^{2}}{|s-t|^{2}} d s d t+\int_{0}^{T} \frac{[u(t)]^{2}}{t} d t .
$$

For $B_{1}: H_{0}^{1}(0, T) \rightarrow L^{2}(0, T)$, we define the adjoint operator $B_{1}^{\prime}: L^{2}(0, T) \rightarrow\left[H_{0}^{1}(0, T)\right]^{\prime}$ via

$$
\left\langle u, B_{1}^{\prime} v\right\rangle_{(0, T)}=\left\langle B_{1} u, v\right\rangle_{L^{2}(0, T)} \quad \text { for all } u \in H_{0,}^{1}(0, T), v \in L^{2}(0, T),
$$

where $\langle\cdot, \cdot\rangle_{(0, T)}$ denotes the duality pairing defined via the extension of the inner product in $L^{2}(0, T)$. Then, we introduce

$$
A:=B_{1}^{\prime} B_{1}: H_{0}^{1}(0, T) \rightarrow\left[H_{0}^{1}(0, T)\right]^{\prime} .
$$

In particular for $u \in H_{0}^{1}(0, T)$, we consider the eigenvalue problem

$$
A u=\lambda u \quad \text { in }\left[H_{0}^{1},(0, T)\right]^{\prime},
$$


i.e., for all $v \in H_{0,}^{1}(0, T)$, we have

$$
\langle A u, v\rangle_{(0, T)}=\left\langle B_{1} u, B_{1} v\right\rangle_{L^{2}(0, T)}=\int_{0}^{T} \partial_{t} u(t) \partial_{t} v(t) d t=\lambda \int_{0}^{T} u(t) v(t) d t .
$$

Note that this is the variational formulation of an eigenvalue problem with mixed boundary conditions,

$$
-\partial_{t t} u(t)=\lambda u(t) \quad \text { for } t \in(0, T), \quad u(0)=0, \quad \partial_{t} u(T)=0 .
$$

Hence, we find

$$
v_{k}(t)=\sin \left(\left(\frac{\pi}{2}+k \pi\right) \frac{t}{T}\right), \quad \lambda_{k}=\frac{1}{T^{2}}\left(\frac{\pi}{2}+k \pi\right)^{2}, \quad k=0,1,2,3, \ldots
$$

Recall that the eigenfunctions $v_{k}$ form an orthogonal basis in $L^{2}(0, T)$ satisfying

$$
\int_{0}^{T} v_{k}(t) v_{\ell}(t) d t=\frac{T}{2} \delta_{k \ell}
$$

and in $H_{0}^{1}(0, T)$,

$$
\int_{0}^{T} \partial_{t} v_{k}(t) \partial_{t} v_{\ell}(t) d t=\lambda_{k} \int_{0}^{T} v_{k}(t) v_{\ell}(t) d t=\frac{1}{2 T}\left(\frac{\pi}{2}+k \pi\right)^{2} \delta_{k \ell} .
$$

For $u \in H_{0}^{1}(0, T)$, this motivates to consider

$$
u(t)=\sum_{k=0}^{\infty} u_{k} \sin \left(\left(\frac{\pi}{2}+k \pi\right) \frac{t}{T}\right), \quad u_{k}=\frac{2}{T} \int_{0}^{T} u(t) \sin \left(\left(\frac{\pi}{2}+k \pi\right) \frac{t}{T}\right) d t
$$

and by Parseval's identity we have

$$
\begin{aligned}
\|u\|_{L^{2}(0, T)}^{2} & =\sum_{k=0}^{\infty} \sum_{\ell=0}^{\infty} u_{k} u_{\ell} \int_{0}^{T} \sin \left(\left(\frac{\pi}{2}+k \pi\right) \frac{t}{T}\right) \sin \left(\left(\frac{\pi}{2}+\ell \pi\right) \frac{t}{T}\right) d t \\
& =\frac{T}{2} \sum_{k=0}^{\infty} u_{k}^{2}
\end{aligned}
$$

as well as

$$
\left\|\partial_{t} u\right\|_{L^{2}(0, T)}^{2}=\sum_{k=0}^{\infty} \sum_{\ell=0}^{\infty} u_{k} u_{\ell} \int_{0}^{T} \partial_{t} v_{k}(t) \partial_{t} v_{\ell}(t) d t=\frac{1}{2 T} \sum_{k=0}^{\infty}\left(\frac{\pi}{2}+k \pi\right)^{2} u_{k}^{2} .
$$

Hence, using interpolation, we define an equivalent norm in $H_{0}^{1 / 2}(0, T)$, e.g., [27, 45],

$$
\|u\|_{H_{0}^{1 / 2}(0, T)}^{2}=\frac{1}{2} \sum_{k=0}^{\infty}\left(\frac{\pi}{2}+k \pi\right) u_{k}^{2}
$$

as well as an inner product

$$
\langle u, v\rangle_{H_{0,}^{1 / 2}(0, T)}=\frac{1}{2} \sum_{k=0}^{\infty}\left(\frac{\pi}{2}+k \pi\right) u_{k} v_{k} .
$$


Analogously, for $w \in H_{, 0}^{1 / 2}(0, T)$ we consider

$$
w(t)=\sum_{k=0}^{\infty} \bar{w}_{k} \cos \left(\left(\frac{\pi}{2}+k \pi\right) \frac{t}{T}\right), \quad \bar{w}_{k}=\frac{2}{T} \int_{0}^{T} w(t) \cos \left(\left(\frac{\pi}{2}+k \pi\right) \frac{t}{T}\right) d t,
$$

with the related norm and inner product,

$$
\|w\|_{H_{, 0}^{1 / 2}(0, T)}^{2}=\frac{1}{2} \sum_{k=0}^{\infty}\left(\frac{\pi}{2}+k \pi\right) \bar{w}_{k}^{2}, \quad\langle w, z\rangle_{H_{, 0}^{1 / 2}(0, T)}=\frac{1}{2} \sum_{k=0}^{\infty}\left(\frac{\pi}{2}+k \pi\right) \bar{w}_{k} \bar{z}_{k} .
$$

Finally, we introduce the dual space $\left[H_{, 0}^{1 / 2}(0, T)\right]^{\prime}$ with the norm

$$
\|f\|_{\left[H_{, 0}^{1 / 2}(0, T)\right]^{\prime}}=\sup _{0 \neq w \in H_{, 0}^{1 / 2}(0, T)} \frac{\langle f, w\rangle_{(0, T)}}{\|w\|_{H_{, 0}^{1 / 2}(0, T)}} .
$$

LEMMA 2.1. For $f \in\left[H_{, 0}^{1 / 2}(0, T)\right]^{\prime}$, we have

$$
\|f\|_{\left[H_{, 0}^{1 / 2}(0, T)\right]^{\prime}}^{2}=\frac{T^{2}}{2} \sum_{k=0}^{\infty}\left(\frac{\pi}{2}+k \pi\right)^{-1} \bar{f}_{k}^{2}
$$

with

$$
\bar{f}_{k}=\frac{2}{T}\left\langle f, w_{k}\right\rangle_{(0, T)}, \quad w_{k}(t)=\cos \left(\left(\frac{\pi}{2}+k \pi\right) \frac{t}{T}\right) .
$$

Proof. From the norm definition, using a series representation of $w \in H_{, 0}^{1 / 2}(0, T)$, and with Hölder's inequality, we have

$$
\begin{aligned}
& \|f\|_{\left[H_{, 0}^{1 / 2}(0, T)\right]^{\prime}} \\
& =\sup _{0 \neq w \in H_{, 0}^{1 / 2}(0, T)} \frac{\langle f, w\rangle_{(0, T)}}{\|w\|_{H_{, 0}^{1 / 2}(0, T)}}=\sup _{0 \neq w \in H_{, 0}^{1 / 2}(0, T)} \frac{\sum_{k=0}^{\infty} \bar{w}_{k}\left\langle f, w_{k}\right\rangle_{(0, T)}}{\left(\frac{1}{2} \sum_{k=0}^{\infty}\left(\frac{\pi}{2}+k \pi\right) \bar{w}_{k}^{2}\right)^{1 / 2}} \\
& =\frac{T}{\sqrt{2}} \sup _{0 \neq w \in H_{, 0}^{1 / 2}(0, T)} \frac{\sum_{k=0}^{\infty} \bar{w}_{k} \bar{f}_{k}}{\left(\sum_{k=0}^{\infty}\left(\frac{\pi}{2}+k \pi\right) \bar{w}_{k}^{2}\right)^{1 / 2}} \leq \frac{T}{\sqrt{2}}\left(\sum_{k=0}^{\infty}\left(\frac{\pi}{2}+k \pi\right)^{-1} \bar{f}_{k}^{2}\right)^{1 / 2},
\end{aligned}
$$

i.e.,

$$
\|f\|_{\left[H_{, 0}^{1 / 2}(0, T)\right]^{\prime}}^{2} \leq \frac{T^{2}}{2} \sum_{k=0}^{\infty}\left(\frac{\pi}{2}+k \pi\right)^{-1} \bar{f}_{k}^{2} .
$$

On the other hand, if the coefficients $\bar{f}_{k}$ are given, we define

$$
\bar{w}_{k}^{*}=\left(\frac{\pi}{2}+k \pi\right)^{-1} \bar{f}_{k}
$$


to prove the opposite direction; we skip the details.

The variational formulation of the initial value problem (2.1) is to find $u \in H_{0,}^{1 / 2}(0, T)$ such that

$$
\left\langle\partial_{t} u, w\right\rangle_{(0, T)}=\langle f, w\rangle_{(0, T)} \quad \text { for all } w \in H_{, 0}^{1 / 2}(0, T),
$$

where $f \in\left[H_{0}^{1 / 2}(0, T)\right]^{\prime}$ is given. Note that (2.7) is a Galerkin-Petrov variational formulation with different trial and test spaces. Hence, we have to establish an appropriate stability condition, which is equivalent to an ellipticity estimate for the bilinear form $\left\langle\partial_{t} u, \mathcal{H}_{T} v\right\rangle_{(0, T)}$ with some transformation operator $\mathcal{H}_{T}: H_{0}^{1 / 2}(0, T) \rightarrow H_{, 0}^{1 / 2}(0, T)$ to be specified.

2.4. Transformation operator. To motivate the particular definition of the operator $\mathcal{H}_{T}: H_{0}^{1 / 2}(0, T) \rightarrow H_{, 0}^{1 / 2}(0, T)$, we write, by using (2.6),

$$
\partial_{t} u(t)=\frac{1}{T} \sum_{k=0}^{\infty} u_{k}\left(\frac{\pi}{2}+k \pi\right) \cos \left(\left(\frac{\pi}{2}+k \pi\right) \frac{t}{T}\right)
$$

as distributional derivative, i.e., for $w \in H_{, 0}^{1 / 2}(0, T)$, we have

$$
\left\langle\partial_{t} u, w\right\rangle_{(0, T)}=\frac{1}{T} \int_{0}^{T} \sum_{k=0}^{\infty} u_{k}\left(\frac{\pi}{2}+k \pi\right) \cos \left(\left(\frac{\pi}{2}+k \pi\right) \frac{t}{T}\right) w(t) d t .
$$

Defining

$$
w(t)=\left(\mathcal{H}_{T} u\right)(t):=\sum_{\ell=0}^{\infty} u_{\ell} \cos \left(\left(\frac{\pi}{2}+\ell \pi\right) \frac{t}{T}\right),
$$

we conclude the ellipticity estimate

$$
\begin{aligned}
\left\langle\partial_{t} u,\right. & \left.\mathcal{H}_{T} u\right\rangle_{(0, T)} \\
& =\frac{1}{T} \sum_{k=0}^{\infty} \sum_{\ell=0}^{\infty} u_{k} u_{\ell}\left(\frac{\pi}{2}+k \pi\right) \int_{0}^{T} \cos \left(\left(\frac{\pi}{2}+k \pi\right) \frac{t}{T}\right) \cos \left(\left(\frac{\pi}{2}+\ell \pi\right) \frac{t}{T}\right) d t \\
& =\frac{1}{2} \sum_{k=0}^{\infty}\left(\frac{\pi}{2}+k \pi\right) u_{k}^{2}=\|u\|_{H_{0,}^{1 / 2}(0, T)}^{2} .
\end{aligned}
$$

REMARK 2.2. The function $\mathcal{H}_{T} u \in H_{, 0}^{1 / 2}(0, T)$, as given in (2.8), is the unique solution of the variational problem

$$
\left\langle\mathcal{H}_{T} u, z\right\rangle_{H_{, 0}^{1 / 2}(0, T)}=\left\langle\partial_{t} u, z\right\rangle_{(0, T)} \quad \text { for all } z \in H_{, 0}^{1 / 2}(0, T) .
$$

Therefore, the definition of the transformation operator $\mathcal{H}_{T}$ coincides with the definition of the optimal test space as used, e.g., in discontinuous Galerkin-Petrov methods [9]. Indeed, for

$$
u(t)=\sum_{k=0}^{\infty} u_{k} \sin \left(\left(\frac{\pi}{2}+k \pi\right) \frac{t}{T}\right)
$$

we use the ansatz

$$
w(t)=\left(\mathcal{H}_{T} u\right)(t)=\sum_{k=0}^{\infty} \bar{w}_{k} \cos \left(\left(\frac{\pi}{2}+k \pi\right) \frac{t}{T}\right)
$$


and the test function

$$
z(t)=\sum_{k=0}^{\infty} \bar{z}_{k} \cos \left(\left(\frac{\pi}{2}+k \pi\right) \frac{t}{T}\right)
$$

to obtain

$$
\langle w, z\rangle_{H_{, 0}^{1 / 2}(0, T)}=\frac{1}{2} \sum_{k=0}^{\infty}\left(\frac{\pi}{2}+k \pi\right) \bar{w}_{k} \bar{z}_{k}=\left\langle\partial_{t} u, z\right\rangle=\frac{1}{2} \sum_{k=0}^{\infty}\left(\frac{\pi}{2}+k \pi\right) u_{k} \bar{z}_{k}
$$

for all $\bar{z}_{k}$, from which we conclude that $\bar{w}_{k}=u_{k}$, for $k=0,1,2, \ldots$

By construction, we have $w=\mathcal{H}_{T} u \in H_{, 0}^{1 / 2}(0, T)$, and $\mathcal{H}_{T}: H_{0}^{1 / 2}(0, T) \rightarrow H_{, 0}^{1 / 2}(0, T)$ is norm preserving, i.e.,

$$
\left\|\mathcal{H}_{T} u\right\|_{H_{, 0}^{1 / 2}(0, T)}=\|u\|_{H_{0,}^{1 / 2}(0, T)} \quad \text { for all } u \in H_{0,}^{1 / 2}(0, T) .
$$

Vice versa, if $w \in H_{, 0}^{1 / 2}(0, T)$ is given,

$$
w(t)=\sum_{k=0}^{\infty} \bar{w}_{k} \cos \left(\left(\frac{\pi}{2}+k \pi\right) \frac{t}{T}\right), \quad \bar{w}_{k}=\frac{2}{T} \int_{0}^{T} w(t) \cos \left(\left(\frac{\pi}{2}+k \pi\right) \frac{t}{T}\right) d t,
$$

then the inverse transformation operator reads

$$
u(t)=\left(\mathcal{H}_{T}^{-1} w\right)(t)=\sum_{k=0}^{\infty} \bar{w}_{k} \sin \left(\left(\frac{\pi}{2}+k \pi\right) \frac{t}{T}\right) .
$$

Next, we are going to prove some properties of the transformation operator $\mathcal{H}_{T}$. First, we consider a commutation property with the time derivative operator $\partial_{t}$.

LEMMA 2.3. For $u \in H_{0}^{1 / 2}(0, T)$, we have

$$
\left\langle\partial_{t} \mathcal{H}_{T} u, v\right\rangle_{(0, T)}=-\left\langle\mathcal{H}_{T}^{-1} \partial_{t} u, v\right\rangle_{(0, T)} \quad \text { for all } v \in H_{0,}^{1 / 2}(0, T) .
$$

Proof. For an arbitrary $\varphi \in C^{\infty}[0, T]$ with $\varphi(0)=0$, we first compute

$$
\left(\mathcal{H}_{T} \varphi\right)(t)=\sum_{k=0}^{\infty} \varphi_{k} \cos \left(\left(\frac{\pi}{2}+k \pi\right) \frac{t}{T}\right), \quad \varphi_{k}=\frac{2}{T} \int_{0}^{T} \varphi(t) \sin \left(\left(\frac{\pi}{2}+k \pi\right) \frac{t}{T}\right) d t
$$

and therefore

$$
\partial_{t}\left(\mathcal{H}_{T} \varphi\right)(t)=-\frac{1}{T} \sum_{k=0}^{\infty} \varphi_{k}\left(\frac{\pi}{2}+k \pi\right) \sin \left(\left(\frac{\pi}{2}+k \pi\right) \frac{t}{T}\right)
$$

follows. On the other hand,

$$
\partial_{t} \varphi(t)=\frac{1}{T} \sum_{k=0}^{\infty} \varphi_{k}\left(\frac{\pi}{2}+k \pi\right) \cos \left(\left(\frac{\pi}{2}+k \pi\right) \frac{t}{T}\right)
$$

implies

$$
\left(\mathcal{H}_{T}^{-1} \partial_{t} \varphi\right)(t)=\frac{1}{T} \sum_{k=0}^{\infty} \varphi_{k}\left(\frac{\pi}{2}+k \pi\right) \sin \left(\left(\frac{\pi}{2}+k \pi\right) \frac{t}{T}\right)
$$


i.e.,

$$
\partial_{t} \mathcal{H}_{T} \varphi=-\mathcal{H}_{T}^{-1} \partial_{t} \varphi \quad \text { for all } \varphi \in C^{\infty}[0, T] \text { with } \varphi(0)=0 .
$$

So, the assertion follows by completion.

Next, we prove that $\mathcal{H}_{T}$ is unitary.

LEMmA 2.4. For $u \in H_{0,}^{1 / 2}(0, T)$ and $w \in H_{, 0}^{1 / 2}(0, T)$, there holds true that

$$
\left\langle\mathcal{H}_{T} u, w\right\rangle_{L^{2}(0, T)}=\left\langle u, \mathcal{H}_{T}^{-1} w\right\rangle_{L^{2}(0, T)} .
$$

Proof. For $u \in H_{0,}^{1 / 2}(0, T)$ and $w \in H_{, 0}^{1 / 2}(0, T)$, we have

$$
u(t)=\sum_{k=0}^{\infty} u_{k} \sin \left(\left(\frac{\pi}{2}+k \pi\right) \frac{t}{T}\right), \quad w(t)=\sum_{\ell=0}^{\infty} \bar{w}_{\ell} \cos \left(\left(\frac{\pi}{2}+\ell \pi\right) \frac{t}{T}\right),
$$

and

$$
\left(\mathcal{H}_{T} u\right)(t)=\sum_{k=0}^{\infty} u_{k} \cos \left(\left(\frac{\pi}{2}+k \pi\right) \frac{t}{T}\right), \quad\left(\mathcal{H}_{T}^{-1} w\right)(t)=\sum_{\ell=0}^{\infty} \bar{w}_{\ell} \sin \left(\left(\frac{\pi}{2}+\ell \pi\right) \frac{t}{T}\right) .
$$

Hence, we compute

$$
\begin{aligned}
\left\langle\mathcal{H}_{T} u, w\right\rangle_{L^{2}(0, T)} & =\int_{0}^{T}\left(\mathcal{H}_{T} u\right)(t) w(t) d t \\
& =\sum_{k=0}^{\infty} \sum_{\ell=0}^{\infty} u_{k} \bar{w}_{\ell} \int_{0}^{T} \cos \left(\left(\frac{\pi}{2}+k \pi\right) \frac{t}{T}\right) \cos \left(\left(\frac{\pi}{2}+\ell \pi\right) \frac{t}{T}\right) d t \\
& =\frac{T}{2} \sum_{k=0}^{\infty} u_{k} \bar{w}_{k} \\
& =\sum_{k=0}^{\infty} \sum_{\ell=0}^{\infty} u_{k} \bar{w}_{\ell} \int_{0}^{T} \sin \left(\left(\frac{\pi}{2}+k \pi\right) \frac{t}{T}\right) \sin \left(\left(\frac{\pi}{2}+\ell \pi\right) \frac{t}{T}\right) d t \\
& =\int_{0}^{T} u(t)\left(\mathcal{H}_{T}^{-1} w\right)(t) d t=\left\langle u, \mathcal{H}_{T}^{-1} w\right\rangle_{L^{2}(0, T)} .
\end{aligned}
$$

Using Lemma 2.3 and Lemma 2.4, we conclude the following symmetry relation.

COROLlary 2.5. For $u, v \in H_{0}^{1 / 2}(0, T)$, there holds true that

$$
\left\langle\partial_{t} u, \mathcal{H}_{T} v\right\rangle_{(0, T)}=\left\langle\mathcal{H}_{T} u, \partial_{t} v\right\rangle_{(0, T)}=\langle u, v\rangle_{H_{0,}^{1 / 2}(0, T)} .
$$

Proof. For $\varphi, \psi \in C^{\infty}[0, T]$ with $\varphi(0)=\psi(0)=0$, we first have $\left(\mathcal{H}_{T} \varphi\right)(T)=$ $\left(\mathcal{H}_{T} \psi\right)(T)=0$, and therefore

$$
\begin{aligned}
\left\langle\partial_{t} \varphi, \mathcal{H}_{T} \psi\right\rangle_{L^{2}(0, T)} & =\left\langle\mathcal{H}_{T}^{-1} \partial_{t} \varphi, \psi\right\rangle_{L^{2}(0, T)} \\
& =-\left\langle\partial_{t} \mathcal{H}_{T} \varphi, \psi\right\rangle_{L^{2}(0, T)} \\
& =-\left.\left(\mathcal{H}_{T} \varphi\right)(t) \psi(t)\right|_{0} ^{T}+\left\langle\mathcal{H}_{T} \varphi, \partial_{t} \psi\right\rangle_{L^{2}(0, T)} \\
& =\left\langle\mathcal{H}_{T} \varphi, \partial_{t} \psi\right\rangle_{L^{2}(0, T)}
\end{aligned}
$$


holds true. So, the assertion follows by completion.

The next property of $\mathcal{H}_{T}$ is required when considering, instead of (2.1), more general differential equations.

LEMMA 2.6. There holds true that

$$
\left\langle v, \mathcal{H}_{T} v\right\rangle_{L^{2}(0, T)} \geq 0 \quad \text { for all } v \in H_{0,}^{1 / 2}(0, T) .
$$

Proof. By using

$$
v(t)=\sum_{k=0}^{\infty} v_{k} \sin \left(\left(\frac{\pi}{2}+k \pi\right) \frac{t}{T}\right), \quad\left(\mathcal{H}_{T} v\right)(t)=\sum_{\ell=0}^{\infty} v_{\ell} \cos \left(\left(\frac{\pi}{2}+\ell \pi\right) \frac{t}{T}\right),
$$

we have

$$
\begin{aligned}
\left\langle v, \mathcal{H}_{T} v\right\rangle_{L^{2}(0, T)} & =\sum_{k=0}^{\infty} \sum_{\ell=0}^{\infty} v_{k} v_{\ell} \int_{0}^{T} \sin \left(\left(\frac{\pi}{2}+k \pi\right) \frac{t}{T}\right) \cos \left(\left(\frac{\pi}{2}+\ell \pi\right) \frac{t}{T}\right) d t \\
& =\frac{1}{2} \sum_{k=0}^{\infty} \sum_{\ell=0}^{\infty} v_{k} v_{\ell} \int_{0}^{T}\left[\sin \left((k+\ell+1) \pi \frac{t}{T}\right)+\sin \left((k-\ell) \pi \frac{t}{T}\right)\right] d t \\
& =\frac{1}{2} \sum_{k=0}^{\infty} \sum_{\ell=0}^{\infty} v_{k} v_{\ell}\left[-\frac{T}{(k+\ell+1) \pi} \cos \left((k+\ell+1) \pi \frac{t}{T}\right)\right]_{0}^{T} \\
& =\frac{T}{2 \pi} \sum_{k=0}^{\infty} \sum_{\ell=0}^{\infty} v_{k} v_{\ell} \frac{1}{k+\ell+1}\left[1-(-1)^{k+\ell+1}\right],
\end{aligned}
$$

where the second integral is ignored due to symmetry. When splitting $k$ and $\ell$ into odd and even indices, i.e., $k=2 i, 2 i+1, \ell=2 j, 2 j+1$, this gives

$$
\begin{aligned}
\left\langle v, \mathcal{H}_{T} v\right\rangle_{L^{2}(0, T)} & =\frac{T}{\pi} \sum_{i=0}^{\infty} \sum_{j=0}^{\infty}\left[\frac{v_{2 i} v_{2 j}}{2 i+2 j+1}+\frac{v_{2 i+1} v_{2 j+1}}{2 i+2 j+3}\right] \\
= & \frac{T}{\pi} \lim _{M \rightarrow \infty} \sum_{i=0}^{M} \sum_{j=0}^{M}\left[v_{2 i} v_{2 j} \int_{0}^{1} x^{2 i+2 j} d x+v_{2 i+1} v_{2 j+1} \int_{0}^{1} x^{2 i+2 j+2} d x\right] \\
= & \frac{T}{\pi} \lim _{M \rightarrow \infty}\left[\int_{0}^{1}\left(\sum_{i=0}^{M} v_{2 i} x^{2 i}\right)^{2} d x+\int_{0}^{1}\left(\sum_{i=0}^{M} v_{2 i+1} x^{2 i+1}\right)^{2} d x\right] \geq 0 .
\end{aligned}
$$

REMARK 2.7. The matrix $H$ as used in the previous proof, i.e.,

$$
H[j, i]=\frac{1}{i+j+1} \quad \text { for } i, j=0,1, \ldots, N,
$$

is a Hilbert matrix [19], which is positive definite but ill-conditioned. For our purpose it is sufficient to use that (2.10) is non-negative.

Next, we will have a closer look at the definition of the transformation operator $\mathcal{H}_{T}$ to see its relation with the well-known Hilbert transform; see, e.g., [20].

LEMMA 2.8. The operator $\mathcal{H}_{T}$ as defined in (2.8) allows the integral representation

$$
\left(\mathcal{H}_{T} u\right)(t)=\text { v.p. } \int_{0}^{T} K(s, t) u(s) d s, \quad t \in(0, T),
$$


as a Cauchy principal value integral, where the kernel function is given as

$$
K(s, t)=\frac{1}{2 T}\left[\frac{1}{\sin \left(\frac{\pi}{2} \frac{s-t}{T}\right)}+\frac{1}{\sin \left(\frac{\pi}{2} \frac{s+t}{T}\right)}\right] .
$$

Proof. Formally, one finds

$$
\begin{aligned}
\left(\mathcal{H}_{T} u\right)(t) & =\sum_{k=0}^{\infty} u_{k} \cos \left(\left(\frac{\pi}{2}+k \pi\right) \frac{t}{T}\right) \\
& =\sum_{k=0}^{\infty} \frac{2}{T} \int_{0}^{T} u(s) \sin \left(\left(\frac{\pi}{2}+k \pi\right) \frac{s}{T}\right) d s \cos \left(\left(\frac{\pi}{2}+k \pi\right) \frac{t}{T}\right) \\
& =\text { v.p. } \int_{0}^{T} u(s) K(s, t) d s
\end{aligned}
$$

with

$$
\begin{aligned}
K(s, t) & =\frac{2}{T} \sum_{k=0}^{\infty} \sin \left(\left(\frac{\pi}{2}+k \pi\right) \frac{s}{T}\right) \cos \left(\left(\frac{\pi}{2}+k \pi\right) \frac{t}{T}\right) \\
& =\frac{1}{T} \sum_{k=0}^{\infty}\left[\sin \left(\left(\frac{\pi}{2}+k \pi\right) \frac{s-t}{T}\right)+\sin \left(\left(\frac{\pi}{2}+k \pi\right) \frac{s+t}{T}\right)\right] .
\end{aligned}
$$

By using the formal representation

$$
\sum_{k=0}^{\infty} \sin \left(\left(\frac{\pi}{2}+k \pi\right) x\right)=\frac{1}{2} \frac{1}{\sin \left(\frac{\pi}{2} x\right)}, \quad \text { for } x \neq 0,2,4, \ldots,
$$

we further conclude the representation (2.11); see [39] for a more detailed proof.

REMARK 2.9. For fixed $s, t \in(0, T), s \neq t$, we consider

$$
\lim _{T \rightarrow \infty} K(s, t)=\frac{1}{\pi} \frac{2 s}{(s-t)(s+t)}
$$

so that

$$
\left(\mathcal{H}_{\infty} u\right)(t)=\text { v.p. } \int_{0}^{\infty} \frac{1}{\pi} \frac{u(s)}{s-t} \frac{2 s}{s+t} d s, \quad t \in(0, \infty),
$$

where the kernel function shows for $s \rightarrow t$ the same behaviour as the Hilbert transform

$$
(\mathcal{H} u)(t)=\text { v.p. } \int_{0}^{\infty} \frac{1}{\pi} \frac{u(s)}{s-t} d s, \quad t \in(0, \infty),
$$

for which all the previous properties are well-known; see, e.g., [20].

2.5. Variational formulations. For the solution of the initial value problem (2.1), we consider the variational formulation (2.7) to find $u \in H_{0,}^{1 / 2}(0, T)$ such that

$$
\left\langle\partial_{t} u, \mathcal{H}_{T} v\right\rangle_{(0, T)}=\left\langle f, \mathcal{H}_{T} v\right\rangle_{(0, T)} \quad \text { for all } v \in H_{0}^{1 / 2}(0, T),
$$




\section{ETNA}

Kent State University and

Johann Radon Institute (RICAM)

where $f \in\left[H_{, 0}^{1 / 2}(0, T)\right]^{\prime}$ is given. Since the bilinear form $\left\langle\partial_{t} u, \mathcal{H}_{T} v\right\rangle_{(0, T)}$ is bounded, i.e., for $u, v \in H_{0,}^{1 / 2}(0, T)$, there holds true that

$$
\left|\left\langle\partial_{t} u, \mathcal{H}_{T} v\right\rangle_{(0, T)}\right| \leq \underbrace{\left\|\partial_{t} u\right\|_{\left[H_{, 0}^{1 / 2}(0, T)\right]^{\prime}}}_{=\left\|B_{1 / 2} u\right\|_{\left[H_{, 0}^{1 / 2}(0, T)\right]^{\prime}}}\left\|\mathcal{H}_{T} v\right\|_{H_{, 0}^{1 / 2}(0, T)}=\|u\|_{H_{0,}^{1 / 2}(0, T)}\|v\|_{H_{0,}^{1 / 2}(0, T)},
$$

and elliptic (see (2.9)), we conclude unique solvability of the variational formulation (2.12).

REMARK 2.10. From the ellipticity estimate (2.9), we also conclude the stability condition

$$
\|u\|_{H_{0,}^{1 / 2}(0, T)}=\frac{\left\langle\partial_{t} u, \mathcal{H}_{T} u\right\rangle_{(0, T)}}{\left\|\mathcal{H}_{T} u\right\|_{H_{, 0}^{1 / 2}(0, T)}} \leq \sup _{0 \neq w \in H_{, 0}^{1 / 2}(0, T)} \frac{\left\langle\partial_{t} u, w\right\rangle_{(0, T)}}{\|w\|_{H_{, 0}^{1 / 2}(0, T)}} \quad \text { for all } u \in H_{0, \quad}^{1 / 2}(0, T),
$$

and from which we conclude unique solvability of the Galerkin-Petrov formulation to find $u \in H_{0,}^{1 / 2}(0, T)$ such that

$$
\left\langle\partial_{t} u, w\right\rangle_{(0, T)}=\langle f, w\rangle_{(0, T)} \quad \text { for all } w \in H_{, 0}^{1 / 2}(0, T)
$$

Next, we consider a conforming finite element discretisation for the variational formulation (2.12). For a time interval $(0, T)$ and a discretisation parameter $N \in \mathbb{N}$, we consider nodes

$$
0=t_{0}<t_{1}<t_{2}<\cdots<t_{N-1}<t_{N}=T
$$

finite elements $\tau_{\ell}=\left(t_{\ell-1}, t_{\ell}\right)$ of local mesh size $h_{\ell}=t_{\ell}-t_{\ell-1}, \ell=1, \ldots, N$, and a related finite element space $S_{h}^{1}(0, T)$ of piecewise linear continuous basis functions $\varphi_{k}, k=0, \ldots, N$, with global mesh size $h=\max _{\ell} h_{\ell}$. Then, the finite element discretisation of the variational formulation (2.12) is to find $u_{h} \in V_{h}:=S_{h}^{1}(0, T) \cap H_{0,}^{1 / 2}(0, T)=\operatorname{span}\left\{\varphi_{k}\right\}_{k=1}^{N}$ such that

$$
\left\langle\partial_{t} u_{h}, \mathcal{H}_{T} v_{h}\right\rangle_{L^{2}(0, T)}=\left\langle f, \mathcal{H}_{T} v_{h}\right\rangle_{(0, T)} \quad \text { for all } v_{h} \in V_{h}
$$

Using standard arguments, e.g., [36], we conclude unique solvability of (2.14) as well as the a priori error estimates

$$
\left\|u-u_{h}\right\|_{H_{0}^{\sigma}(0, T)} \leq c h^{s-\sigma}\|u\|_{H^{s}(0, T)}
$$

when assuming $u \in H^{s}(0, T)$ for some $s \in[1,2]$ and for $\sigma=0, \frac{1}{2}, 1$. Note that for $\sigma=\frac{1}{2}$, the estimate (2.15) is a consequence of Céa's lemma and the approximation property of $S_{h}^{1}(0, T)$, while for $\sigma=0$ we use the Aubin-Nitsche trick, and for $\sigma=1$, we have to use an inverse inequality, i.e., we have to assume a globally quasi-uniform mesh in this case.

The Galerkin-Bubnov finite element formulation (2.14) is equivalent to the linear system of algebraic equations $K_{h} \underline{u}=\underline{f}$ with a symmetric and positive definite stiffness matrix $K_{h}$ defined by

$$
K_{h}[j, k]=\left\langle\partial_{t} \varphi_{k}, \mathcal{H}_{T} \varphi_{j}\right\rangle_{L^{2}(0, T)} \quad \text { for } k, j=1, \ldots, N
$$

As a numerical example, we consider the solution $u(t)=\sin \left(\frac{9 \pi}{4} t\right)$ for $t \in(0,2)=(0, T)$, where the right-hand side is $f(t)=\frac{9 \pi}{4} \cos \left(\frac{9 \pi}{4} t\right)$. For the discretisation, we consider a sequence of finite element spaces $S_{h}^{1}(0, T)$ of uniform mesh size $h=2 / N$, and $N=2^{j+1}$, $j=0, \ldots, 7$. Since the solution $u$ is smooth, we use $s=2$ within the error estimate (2.15) to 
conclude second-order convergence in $L^{2}(0,2)$ and linear convergence in $H^{1}(0,2)$, respectively. This behaviour is confirmed by the numerical results given in Table 2.1. In addition, we present the minimal and maximal eigenvalues of the stiffness matrix $K_{h}$ as well as the resulting spectral condition number of $K_{h}$, which behave as expected for a first-order differential operator. Note that these results correspond to the Galerkin discretisation of a hypersingular boundary integral operator in boundary element methods for second-order elliptic partial differential equations; see, e.g., [36].

TABLE 2.1

Numerical results for the Galerkin-Bubnov formulation (2.14).

\begin{tabular}{rccccccr}
$\mathrm{N}$ & $\left\|u-u_{h}\right\|_{L^{2}}$ & eoc & $\left\|\partial_{t}\left(u-u_{h}\right)\right\|_{L^{2}}$ & eoc & $\lambda_{\min }\left(K_{h}\right)$ & $\lambda_{\max }\left(K_{h}\right)$ & $\kappa_{2}\left(K_{h}\right)$ \\
\hline 2 & 1.00473818 & - & 7.05949197 & - & 0.4166 & 0.9602 & 2.3 \\
4 & 0.86127822 & 0.2 & 5.88004588 & 0.3 & 0.2844 & 1.1169 & 3.9 \\
8 & 0.16924553 & 2.3 & 3.66044528 & 0.7 & 0.1688 & 1.1280 & 6.7 \\
16 & 0.03246999 & 2.4 & 1.82612730 & 1.0 & 0.0915 & 1.1327 & 12.4 \\
32 & 0.00748649 & 2.1 & 0.90514235 & 1.0 & 0.0475 & 1.1338 & 23.9 \\
64 & 0.00183184 & 2.0 & 0.45124173 & 1.0 & 0.0241 & 1.1340 & 47.0 \\
128 & 0.00045545 & 2.0 & 0.22543481 & 1.0 & 0.0122 & 1.1341 & 93.2 \\
256 & 0.00011371 & 2.0 & 0.11269290 & 1.0 & 0.0061 & 1.1341 & 185.6
\end{tabular}

The evaluation of the transformed basis functions $\mathcal{H}_{T} \varphi_{k}$ can be done by using the definition (2.8). Although the piecewise linear basis functions $\varphi_{k}$ have local support, the transformed basis functions $\mathcal{H}_{T} \varphi_{k}$ are global (see Figure 2.1), and therefore the stiffness matrix $K_{h}$ is dense. As in the case of the hypersingular boundary integral operator, one may use different techniques such as adaptive cross approximation [33] to accelerate the computations, but this is far beyond the scope of this contribution; see Remark 3.6.

Instead of the initial value problem (2.1), for $\mu>0$, we consider the first-order ordinary differential equation

$$
\partial_{t} u(t)+\mu u(t)=f(t) \quad \text { for } t \in(0, T), \quad u(0)=0,
$$

and the related variational formulation to find $u \in H_{0}^{1 / 2}(0, T)$ such that

$$
\left\langle\partial_{t} u, \mathcal{H}_{T} v\right\rangle_{(0, T)}+\mu\left\langle u, \mathcal{H}_{T} v\right\rangle_{L^{2}(0, T)}=\left\langle f, \mathcal{H}_{T} v\right\rangle_{(0, T)} \quad \text { for all } v \in H_{0,}^{1 / 2}(0, T),
$$

where $f \in\left[H_{, 0}^{1 / 2}(0, T)\right]^{\prime}$ is given. When combining (2.9) and (2.10), this gives

$$
\left\langle\partial_{t} v, \mathcal{H}_{T} v\right\rangle_{(0, T)}+\mu\left\langle v, \mathcal{H}_{T} v\right\rangle_{L^{2}(0, T)} \geq\left\langle\partial_{t} v, \mathcal{H}_{T} v\right\rangle_{(0, T)}=\|v\|_{H_{0,}^{1 / 2}(0, T)}^{2}
$$

for all $v \in H_{0}^{1 / 2}(0, T)$, i.e., the bilinear form of the variational problem (2.17) is bounded and elliptic, implying unique solvability of (2.17). For the solution $u \in H_{0,}^{1 / 2}(0, T)$ of the variational problem (2.17), we have

$$
\begin{aligned}
\|u\|_{H_{0,}^{1 / 2}(0, T)}^{2} & =\left\langle\partial_{t} u, \mathcal{H}_{T} u\right\rangle_{(0, T)} \leq\left\langle\partial_{t} u, \mathcal{H}_{T} u\right\rangle_{(0, T)}+\mu\left\langle u, \mathcal{H}_{T} u\right\rangle_{L^{2}(0, T)} \\
& =\left\langle f, \mathcal{H}_{T} u\right\rangle_{(0, T)} \leq\|f\|_{\left[H_{, 0}^{1 / 2}(0, T)\right]^{\prime}}\left\|\mathcal{H}_{T} u\right\|_{H_{, 0}^{1 / 2}(0, T)},
\end{aligned}
$$

implying

$$
\|u\|_{H_{0,}^{1 / 2}(0, T)} \leq\|f\|_{\left[H_{0}^{1 / 2}(0, T)\right]^{\prime}} .
$$



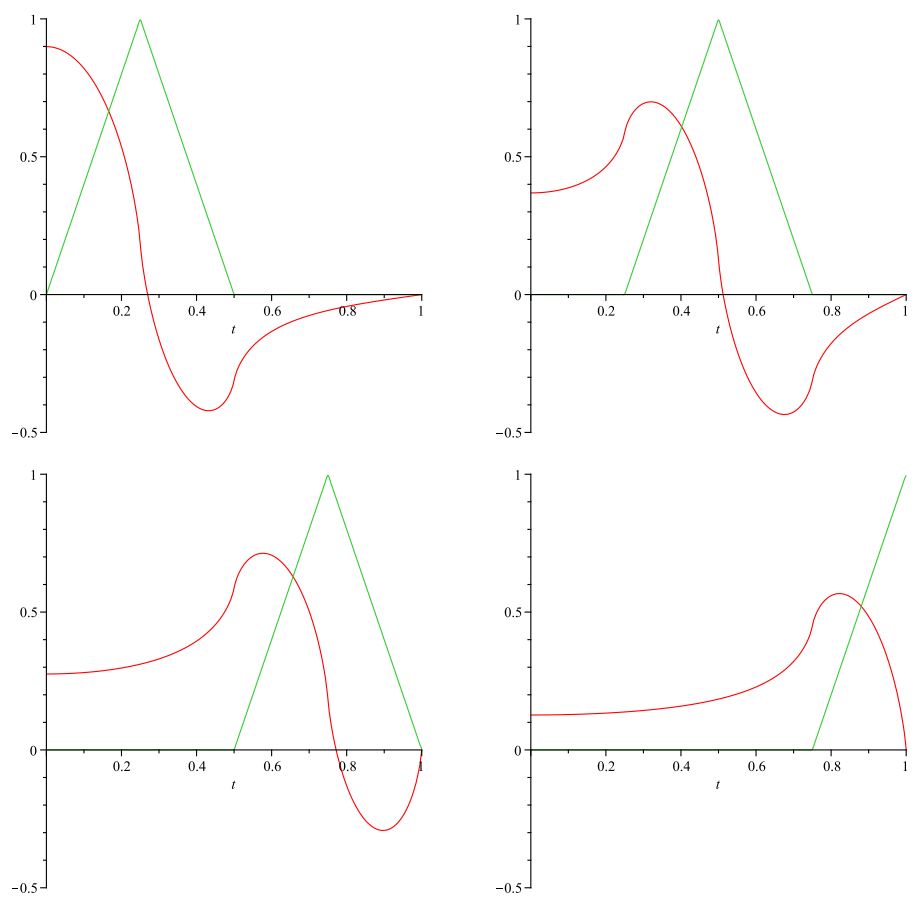

FIG. 2.1. Transformed basis functions $\mathcal{H}_{T} \varphi_{k}, k=1, \ldots, N, N=4$.

For the analysis of the heat equation, we also need to have appropriate estimates for the solution $u$ in $L^{2}(0, T)$.

LEMMA 2.11. Let $u \in H_{0}^{1 / 2}(0, T)$ be the unique solution of the variational problem (2.17), where $f \in\left[H_{, 0}^{1 / 2}(0, T)\right]^{\prime}$ is given. Then,

$$
\|u\|_{L^{2}(0, T)}^{2} \leq \frac{T}{2} \sum_{k=0}^{\infty} \frac{\bar{f}_{k}^{2}}{\mu^{2}+\frac{1}{T^{2}}\left(\frac{\pi}{2}+k \pi\right)^{2}},
$$

where

$$
\bar{f}_{k}:=\frac{2}{T}\left\langle f, w_{k}\right\rangle_{(0, T)}, \quad w_{k}(t):=\cos \left(\left(\frac{\pi}{2}+k \pi\right) \frac{t}{T}\right) .
$$

Proof. Let $\left(f_{n}\right)_{n \in \mathbb{N}} \subset L^{2}(0, T)$ be a sequence with $\lim _{n \rightarrow \infty}\left\|f-f_{n}\right\|_{\left[H_{, 0}^{1 / 2}(0, T)\right]^{\prime}}=0$. We write $f_{n} \in L^{2}(0, T)$ as

$$
\begin{aligned}
f_{n}(t) & =\sum_{k=0}^{\infty} \bar{f}_{n, k} \cos \left(\left(\frac{\pi}{2}+k \pi\right) \frac{t}{T}\right), \\
\bar{f}_{n, k} & =\frac{2}{T} \int_{0}^{T} f_{n}(t) \cos \left(\left(\frac{\pi}{2}+k \pi\right) \frac{t}{T}\right) d t .
\end{aligned}
$$

Let $u_{n} \in H_{0}^{1 / 2}(0, T)$ be the weak solution of the differential equation (2.16) with right-hand side $f_{n}$. It follows analogously to (2.18) that

$$
\left\|u-u_{n}\right\|_{H_{0,}^{1 / 2}(0, T)} \leq\left\|f-f_{n}\right\|_{\left[H_{, 0}^{1 / 2}(0, T)\right]^{\prime}}
$$


and therefore $u_{n} \rightarrow u$ in $H_{0}^{1 / 2}(0, T)$ and $u_{n} \rightarrow u$ in $L^{2}(0, T)$ as $n \rightarrow \infty$.

Because of $f_{n} \in L^{2}(0, T)$ and using (2.20), we have the representation

$$
\begin{aligned}
u_{n}(t) & =\int_{0}^{t} e^{\mu(s-t)} f_{n}(s) d s=\sum_{k=0}^{\infty} \bar{f}_{n, k} \int_{0}^{t} e^{\mu s} \cos \left(a_{k} s\right) d s e^{-\mu t} \\
& =\sum_{k=0}^{\infty} \frac{\bar{f}_{n, k}}{\mu^{2}+a_{k}^{2}}\left[a_{k} \sin \left(a_{k} t\right)+\mu \cos \left(a_{k} t\right)-\mu e^{-\mu t}\right], \quad a_{k}=\frac{1}{T}\left(\frac{\pi}{2}+k \pi\right),
\end{aligned}
$$

and we obtain, when computing all integrals, that

$$
\left\|u_{n}\right\|_{L^{2}(0, T)}^{2}=\frac{T}{2} \sum_{k=0}^{\infty} \frac{\bar{f}_{n, k}^{2}}{\mu^{2}+a_{k}^{2}}-\frac{1}{2} \mu\left[1+e^{-2 \mu T}\right]\left(\sum_{k=0}^{\infty} \frac{\bar{f}_{n, k}}{\mu^{2}+a_{k}^{2}}\right)^{2} \leq \frac{T}{2} \sum_{k=0}^{\infty} \frac{\bar{f}_{n, k}^{2}}{\mu^{2}+a_{k}^{2}} .
$$

So, the assertion follows as $n \rightarrow \infty$.

REMARK 2.12. From (2.19), we immediately conclude the estimate

$$
\|u\|_{L^{2}(0, T)}^{2} \leq \frac{T^{3}}{2} \sum_{k=0}^{\infty}\left(\frac{\pi}{2}+k \pi\right)^{-2} \bar{f}_{k}^{2}=\|f\|_{\left[H_{, 0}^{1}(0, T)\right]^{\prime}}^{2} .
$$

Moreover, when we assume $f \in L^{2}(0, T)$, inequality (2.19) gives

$$
\|u\|_{L^{2}(0, T)}^{2} \leq \frac{T}{2 \mu^{2}} \sum_{k=0}^{\infty} \bar{f}_{k}^{2}=\frac{1}{\mu^{2}}\|f\|_{L^{2}(0, T)}^{2}, \quad \text { i.e., } \quad \mu\|u\|_{L^{2}(0, T)} \leq\|f\|_{L^{2}(0, T)} .
$$

The Galerkin-Bubnov discretisation of (2.17) is to find $u_{h} \in V_{h}$ such that

(2.21) $\left\langle\partial_{t} u_{h}, \mathcal{H}_{T} v_{h}\right\rangle_{L^{2}(0, T)}+\mu\left\langle u_{h}, \mathcal{H}_{T} v_{h}\right\rangle_{L^{2}(0, T)}=\left\langle f, \mathcal{H}_{T} v_{h}\right\rangle_{(0, T)} \quad$ for all $v_{h} \in V_{h}$.

As for the initial value problem (2.1), we have unique solvability of (2.21), but related a priori error estimates depend on $\mu$ in general, requiring a sufficient small mesh size $h$ to ensure convergence for large $\mu$.

REMARK 2.13. Instead of the Galerkin-Bubnov variational formulation (2.17), we may also consider the Galerkin-Petrov formulation to find $u \in H_{0}^{1 / 2}(0, T)$ such that

$$
\left\langle\partial_{t} u, w\right\rangle_{(0, T)}+\mu\langle u, w\rangle_{L^{2}(0, T)}=\langle f, w\rangle_{(0, T)} \quad \text { for all } w \in H_{, 0}^{1 / 2}(0, T),
$$

where the ellipticity of $\left\langle\partial_{t} v, \mathcal{H}_{T} v\right\rangle_{(0, T)}+\mu\left\langle v, \mathcal{H}_{T} v\right\rangle_{L^{2}(0, T)}$ implies a related stability estimate, from which unique solvability of (2.22) follows.

For the finite element discretisation of the Galerkin-Petrov variational formulations (2.13) and (2.22), we have to define a suitable test space $W_{h} \subset H_{, 0}^{1 / 2}(0, T)$. A first choice is to use $W_{h}:=S_{h}^{1}(0, T) \cap H_{, 0}^{1 / 2}(0, T)$. Although the discrete systems are always uniquely solvable since the stiffness matrices are regular lower triangular, the resulting scheme is never stable when considering (2.16). The construction of a more suitable test space is, in particular when considering partial differential equations such as the heat equation, more challenging.

3. The heat equation. As model problem for a parabolic partial differential equation, we consider the Dirichlet problem for the heat equation,

$$
\begin{aligned}
\partial_{t} u(x, t)-\Delta_{x} u(x, t) & =f(x, t) & & \text { for }(x, t) \in Q:=\Omega \times(0, T), \\
u(x, t) & =0 & & \text { for }(x, t) \in \Sigma:=\Gamma \times(0, T), \\
u(x, 0) & =0 & & \text { for } x \in \Omega,
\end{aligned}
$$


where $\Omega \subset \mathbb{R}^{d}, d=1,2,3$, is a bounded domain with, for $d=2,3$, Lipschitz boundary $\Gamma=\partial \Omega$. To write down a variational formulation, we need to have suitable Sobolev spaces. In addition to the eigenfunctions $v_{k}(t)$ and eigenvalues $\lambda_{k}$ as given in (2.5), we consider the eigenfunctions $\phi_{i} \in H_{0}^{1}(\Omega)$ and the associated eigenvalues $\mu_{i}, i \in \mathbb{N}$, of the spatial Dirichlet eigenvalue problem

$$
-\Delta_{x} \phi=\mu \phi \quad \text { in } \Omega, \quad \phi=0 \quad \text { on } \Gamma, \quad\|\phi\|_{L^{2}(\Omega)}=1 .
$$

Recall that the eigenfunctions $\phi_{i}$ form an orthonormal basis in $L^{2}(\Omega)$ and an orthogonal basis in $H_{0}^{1}(\Omega)$. In addition, we have

$$
0<\mu_{1} \leq \mu_{2} \leq \mu_{3} \leq \ldots \quad \text { and } \quad \mu_{i} \rightarrow \infty \quad \text { as } \rightarrow \infty .
$$

Therefore, for a function $u \in L^{2}(Q)$, we find the representation

$$
u(x, t)=\sum_{i=1}^{\infty} \sum_{k=0}^{\infty} u_{i, k} v_{k}(t) \phi_{i}(x)=\sum_{i=1}^{\infty} U_{i}(t) \phi_{i}(x), \quad U_{i}(t)=\sum_{k=0}^{\infty} u_{i, k} v_{k}(t)
$$

with the coefficients

$$
\begin{aligned}
u_{i, k} & =\frac{2}{T} \int_{0}^{T} \int_{\Omega} u(x, t) v_{k}(t) \phi_{i}(x) d x d t \\
& =\frac{2}{T} \int_{0}^{T} \sin \left(\left(\frac{\pi}{2}+k \pi\right) \frac{t}{T}\right) \int_{\Omega} u(x, t) \phi_{i}(x) d x d t
\end{aligned}
$$

Note that we have

$$
\|u\|_{L^{2}(Q)}^{2}=\sum_{i=1}^{\infty}\left\|U_{i}\right\|_{L^{2}(0, T)}^{2}=\frac{T}{2} \sum_{i=1}^{\infty} \sum_{k=0}^{\infty} u_{i, k}^{2}
$$

and

$$
\begin{aligned}
|u|_{H^{1}(Q)}^{2} & =\sum_{i=1}^{\infty}\left[\left\|\partial_{t} U_{i}\right\|_{L^{2}(0, T)}^{2}+\mu_{i}\left\|U_{i}\right\|_{L^{2}(0, T)}^{2}\right] \\
& =\frac{T}{2} \sum_{i=1}^{\infty} \sum_{k=0}^{\infty}\left[\frac{1}{T^{2}}\left(\frac{\pi}{2}+k \pi\right)^{2}+\mu_{i}\right] u_{i, k}^{2} .
\end{aligned}
$$

This motivates to define the norm, for $u \in H^{1}(Q)$ with $u(\cdot, 0)=u_{\mid \Sigma}=0$,

$$
\begin{aligned}
\|u\|_{H_{0 ; 0}^{1,1 / 2}(Q)}^{2} & :=\sum_{i=1}^{\infty}\left[\left\|U_{i}\right\|_{H_{0,}^{1 / 2}(0, T)}^{2}+\mu_{i}\left\|U_{i}\right\|_{L^{2}(0, T)}^{2}\right] \\
& =\frac{T}{2} \sum_{i=1}^{\infty} \sum_{k=0}^{\infty}\left[\frac{1}{T}\left(\frac{\pi}{2}+k \pi\right)+\mu_{i}\right] u_{i, k}^{2}
\end{aligned}
$$

and to introduce the anisotropic Sobolev space

$$
H_{0 ; 0,}^{1,1 / 2}(Q):=\left\{u \in L^{2}(Q):\|u\|_{H_{0 ; 0}^{1,1 / 2}(Q)}<\infty\right\}
$$


Note that $H_{0 ; 0}^{1,1 / 2}(Q)=H_{0,}^{1 / 2}\left(0, T ; L^{2}(\Omega)\right) \cap L^{2}\left(0, T ; H_{0}^{1}(\Omega)\right)$. Analogously, we introduce $H_{0 ;, 0}^{1,1 / 2}(Q)=H_{, 0}^{1 / 2}\left(0, T ; L^{2}(\Omega)\right) \cap L^{2}\left(0, T ; H_{0}^{1}(\Omega)\right)$, which is equipped with the norm

$$
\|w\|_{H_{0 ;, 0}^{1,1 / 2}(Q)}^{2}=\frac{T}{2} \sum_{i=1}^{\infty} \sum_{k=0}^{\infty}\left[\frac{1}{T}\left(\frac{\pi}{2}+k \pi\right)+\mu_{i}\right] \bar{w}_{i, k}^{2}
$$

and

$$
\bar{w}_{i, k}=\frac{2}{T} \int_{0}^{T} \cos \left(\left(\frac{\pi}{2}+k \pi\right) \frac{t}{T}\right) \int_{\Omega} w(x, t) \phi_{i}(x) d x d t .
$$

LEMMA 3.1. For the dual norm of $f \in\left[H_{0 ; 0}^{1,1 / 2}(Q)\right]^{\prime}$, we have

$$
\|f\|_{\left[H_{0,0}^{1,1 / 2}(Q)\right]^{\prime}}^{2}=\frac{T}{2} \sum_{i=1}^{\infty} \sum_{k=0}^{\infty}\left[\frac{1}{T}\left(\frac{\pi}{2}+k \pi\right)+\mu_{i}\right]^{-1} \bar{f}_{i, k}^{2}
$$

with

$$
\bar{f}_{i, k}=\frac{2}{T}\left\langle f, w_{k} \phi_{i}\right\rangle_{Q}
$$

Proof. First, from the norm definition, using a series representation of $w \in H_{0 ;, 0}^{1,1 / 2}(Q)$, and with Hölder's inequality, we have

$$
\begin{aligned}
& \|f\|_{\left[H_{0 ;, 0}^{1,1 / 2}(Q)\right]^{\prime}}=\sup _{0 \neq w \in H_{0 ;, 0}^{1,1}(Q)} \frac{\langle f, w\rangle_{Q}}{\|w\|_{H_{0 ;, 0}^{1,1 / 2}(Q)}} \\
= & \sup _{0 \neq w \in H_{0 ;, 0}^{1,1 / 2}(Q)} \frac{\sum_{i=1}^{\infty} \sum_{k=0}^{\infty} \bar{w}_{i, k}\left\langle f, w_{k} \phi_{i}\right\rangle_{Q}}{\left(\frac{T}{2} \sum_{i=1}^{\infty} \sum_{k=0}^{\infty}\left[\frac{1}{T}\left(\frac{\pi}{2}+k \pi\right)+\mu_{i}\right] \bar{w}_{i, k}^{2}\right)^{1 / 2}} \\
= & \frac{\sqrt{T}}{\sqrt{2}} \sup _{0 \neq w \in H_{0 ;, 0}^{1,1 / 2}(Q)} \frac{\sum_{i=1}^{\infty} \sum_{k=0}^{\infty} \bar{w}_{i, k} \bar{f}_{i, k}}{\left(\sum_{i=1}^{\infty} \sum_{k=0}^{\infty}\left[\frac{1}{T}\left(\frac{\pi}{2}+k \pi\right)+\mu_{i}\right] \bar{w}_{i, k}^{2}\right)^{1 / 2}} \\
\leq & \frac{\sqrt{T}}{\sqrt{2}}\left(\sum_{i=1}^{\infty} \sum_{k=0}^{\infty}\left[\frac{1}{T}\left(\frac{\pi}{2}+k \pi\right)+\mu_{i}\right]^{-1} \bar{f}_{i, k}^{2}\right)^{1 / 2},
\end{aligned}
$$

i.e.,

$$
\|f\|_{\left[H_{0 ;, 0}^{1,1 / 2}(Q)\right]^{\prime}}^{2} \leq \frac{T}{2} \sum_{i=1}^{\infty} \sum_{k=0}^{\infty}\left[\frac{1}{T}\left(\frac{\pi}{2}+k \pi\right)+\mu_{i}\right]^{-1} \bar{f}_{i, k}^{2} .
$$

The lower estimate follows as in the proof of Lemma 2.1; we skip the details.

According to the previous sections, we consider the variational formulation of (3.1) to find $u \in H_{0 ; 0}^{1,1 / 2}(Q)$ such that

$$
\left\langle\partial_{t} u, v\right\rangle_{Q}+\left\langle\nabla_{x} u, \nabla_{x} v\right\rangle_{L^{2}(Q)}=\langle f, v\rangle_{Q}
$$


is satisfied for all $v \in H_{0 ;, 0}^{1,1 / 2}(Q)$, where $f \in\left[H_{0 ;, 0}^{1,1 / 2}(Q)\right]^{\prime}$ is given, and $\langle\cdot, \cdot\rangle_{Q}$ denotes the duality pairing as the extension of the inner product in $L^{2}(Q)$. For the following result, see also [4, Lemma 2.8], [27, 28], and [35, Corollary 3.9].

THEOREM 3.2. The variational formulation (3.3) implies an isomorphism

$$
\mathcal{L}: H_{0 ; 0}^{1,1 / 2}(Q) \rightarrow\left[H_{0 ;, 0}^{1,1 / 2}(Q)\right]^{\prime},
$$

satisfying

$$
\|u\|_{H_{0 ; 0}^{1,1 / 2}(Q)} \leq 2\|\mathcal{L} u\|_{\left[H_{0 ; 0}^{1,1 / 2}(Q)\right]^{\prime}} \quad \text { for all } u \in H_{0 ; 0,}^{1,1 / 2}(Q) .
$$

Proof. For the solution $u$ of the variational problem (3.3), we use the ansatz (3.2), where $U_{i} \in H_{0}^{1 / 2}(0, T)$ are unknown functions to be determined. When choosing as test function $v(x, t):=V(t) \phi_{j}(x)$ for a fixed $j \in \mathbb{N}$ with $V \in H_{, 0}^{1 / 2}(0, T)$, the variational formulation (3.3) leads to find $U_{j} \in H_{0}^{1 / 2}(0, T)$ such that

$$
\left\langle\partial_{t} U_{j}, V\right\rangle_{(0, T)}+\mu_{j}\left\langle U_{j}, V\right\rangle_{L^{2}(0, T)}=\left\langle f, V \phi_{j}\right\rangle_{Q}
$$

is satisfied for all $V \in H_{, 0}^{1 / 2}(0, T)$. It holds true that

$\left|\left\langle f, V \phi_{j}\right\rangle_{Q}\right| \leq\|f\|_{\left[H_{0 ;, 0}^{1,1 / 2}(Q)\right]^{\prime}}\left\|V \phi_{j}\right\|_{H_{0 ;, 0}^{1,1 / 2}(Q)} \leq \sqrt{1+\frac{T}{\sqrt{2}} \mu_{j}}\|f\|_{\left[H_{0 ;, 0}^{1,1 / 2}(Q)\right]^{\prime}}\|V\|_{H_{, 0}^{1 / 2}(0, T)}$

for all $V \in H_{, 0}^{1 / 2}(0, T)$, and so $\left\langle f_{j}, V\right\rangle_{(0, T)}:=\left\langle f, V \phi_{j}\right\rangle_{Q}$ fulfils $f_{j} \in\left[H_{, 0}^{1 / 2}(0, T)\right]^{\prime}$. The unique solvability of (3.5) follows analogously as for (2.16). So, for every $j \in \mathbb{N}$, we have a unique solution $U_{j} \in H_{0}^{1 / 2}(0, T)$ of the variational formulation (3.5) satisfying

$$
\begin{aligned}
\left\|U_{j}\right\|_{H_{0,}^{1 / 2}(0, T)}^{2} & =\left\langle\partial_{t} U_{j}, \mathcal{H}_{T} U_{j}\right\rangle_{(0, T)} \\
& \leq\left\langle\partial_{t} U_{j}, \mathcal{H}_{T} U_{j}\right\rangle_{(0, T)}+\mu_{j}\left\langle U_{j}, \mathcal{H}_{T} U_{j}\right\rangle_{L^{2}(0, T)} \\
& =\left\langle f, \phi_{j} \mathcal{H}_{T} U_{j}\right\rangle_{Q} .
\end{aligned}
$$

For $M \in \mathbb{N}$, we define

$$
u_{M}(x, t)=\sum_{j=1}^{M} U_{j}(t) \phi_{j}(x)
$$

and we conclude that

$$
\begin{aligned}
\left\|u_{M}\right\|_{H_{0,}^{1 / 2}\left(0, T ; L^{2}(\Omega)\right)}^{2} & =\sum_{j=1}^{M}\left\|U_{j}\right\|_{H_{0}^{1 / 2}(0, T)}^{2} \leq \sum_{j=1}^{M}\left\langle f, \phi_{j} \mathcal{H}_{T} U_{j}\right\rangle_{Q} \\
& =\left\langle f, \mathcal{H}_{T} u_{M}\right\rangle_{Q} \\
& \leq\|f\|_{\left[H_{0 ;, 0}^{1,1 / 2}(Q)\right]^{\prime}}\left\|\mathcal{H}_{T} u_{M}\right\|_{H_{0 ;, 0}^{1,1 / 2}(Q)} \\
& =\|f\|_{\left[H_{0 ;, 0}^{1,1 / 2}(Q)\right]^{\prime}}\left\|u_{M}\right\|_{H_{0 ; 0}^{1,1 / 2}(Q)} .
\end{aligned}
$$

Hence, using (2.19) for

$$
\bar{f}_{i, k}=\frac{2}{T}\left\langle f_{i}, w_{k}\right\rangle_{(0, T)}=\frac{2}{T}\left\langle f, \phi_{i} w_{k}\right\rangle_{Q}
$$


we obtain

$$
\begin{aligned}
\left\|u_{M}\right\|_{L^{2}\left(0, T ; H_{0}^{1}(\Omega)\right)}^{2} & =\sum_{i=1}^{M} \mu_{i}\left\|U_{i}\right\|_{L^{2}(0, T)}^{2} \\
& \leq \frac{T}{2} \sum_{i=1}^{M} \sum_{k=0}^{\infty} \frac{\mu_{i}}{\mu_{i}^{2}+\frac{1}{T^{2}}\left(\frac{\pi}{2}+k \pi\right)^{2}} \bar{f}_{i, k}^{2} \\
& \leq T \sum_{i=1}^{M} \sum_{k=0}^{\infty} \frac{1}{\mu_{i}+\frac{1}{T}\left(\frac{\pi}{2}+k \pi\right)} \bar{f}_{i, k}^{2} \leq 2\|f\|_{\left[H_{0 ; 0}^{1,1 / 2}(Q)\right]^{\prime}}^{2},
\end{aligned}
$$

where we have used

$$
\frac{a}{a^{2}+b^{2}} \leq \frac{a+b}{\frac{1}{2}(a+b)^{2}}=\frac{2}{a+b} \quad \text { for } 0<a, b \in \mathbb{R} .
$$

With this, we have

$$
\begin{aligned}
\left\|u_{M}\right\|_{H_{0 ; 0}^{1,1 / 2}(Q)}^{2} & =\left\|u_{M}\right\|_{H_{0,}^{1 / 2}\left(0, T ; L^{2}(Q)\right)}^{2}+\left\|u_{M}\right\|_{L^{2}\left(0, T ; H_{0}^{1}(\Omega)\right)}^{2} \\
& \leq\|f\|_{\left[H_{0 ; 0}^{1,1 / 2}(Q)\right]^{\prime}}\left\|u_{M}\right\|_{H_{0 ; 0}^{1,1 / 2}(Q)}+2\|f\|_{\left[H_{0 ; 0}^{1,1 / 2}(Q)\right]^{\prime}}^{2}
\end{aligned}
$$

and therefore

$$
\left\|u_{M}\right\|_{H_{0 ; 0,}^{1,1 / 2}(Q)} \leq 2\|f\|_{\left[H_{0 ;, 0}^{1,1 / 2}(Q)\right]^{\prime}}
$$

follows for all $M \in \mathbb{N}$. The last inequality yields the bound

$$
\begin{aligned}
\|u\|_{H_{0 ; 0}^{1,1 / 2}(Q)}^{2} & =\lim _{M \rightarrow \infty} \sum_{i=1}^{M}\left[\left\|U_{i}\right\|_{H_{0,}^{1 / 2}(0, T)}^{2}+\mu_{i}\left\|U_{i}\right\|_{L^{2}(0, T)}^{2}\right] \\
& =\lim _{M \rightarrow \infty}\left\|u_{M}\right\|_{H_{0 ; 0}^{1,1 / 2}(Q)}^{2} \leq 4\|f\|_{\left[H_{0 ; 0}^{1,1 / 2}(Q)\right]^{\prime}}^{2}<\infty
\end{aligned}
$$

and thus, $u \in H_{0 ; 0,}^{1,1 / 2}(Q)$ with $\lim _{M \rightarrow \infty} u_{M}=u$ in $H_{0 ; 0,}^{1,1 / 2}(Q)$.

The existence of a solution of the variational formulation (3.3) is proven by inserting the constructed function $u$ into the variational formulation (3.3) and using the approximating sequence $\left(u_{M}\right)_{M \in \mathbb{N}}$. The uniqueness of a solution of the variational formulation (3.3) is a consequence of the uniqueness of the coefficient functions $U_{j}$.

COROLLARY 3.3. As a direct consequence of (3.4), we immediately conclude the stability estimate

$$
\frac{1}{2}\|u\|_{H_{0 ; 0}^{1,1 / 2}(Q)} \leq \sup _{0 \neq w \in H_{0 ;, 0}^{1,1 / 2}(Q)} \frac{\left\langle\partial_{t} u, w\right\rangle_{Q}+\left\langle\nabla_{x} u, \nabla_{x} w\right\rangle_{L^{2}(Q)}}{\|w\|_{H_{0 ;, 0}^{1,1 / 2}(Q)}}
$$

for all $u \in H_{0 ; 0,}^{1,1 / 2}(Q)$.

The variational formulation (3.3) is equivalent to find $u \in H_{0 ; 0,}^{1,1 / 2}(Q)$ such that

$$
\left\langle\partial_{t} u, \mathcal{H}_{T} v\right\rangle_{Q}+\left\langle\nabla_{x} u, \nabla_{x} \mathcal{H}_{T} v\right\rangle_{L^{2}(Q)}=\left\langle f, \mathcal{H}_{T} v\right\rangle_{Q}
$$

is satisfied for all $v \in H_{0 ; 0,}^{1,1 / 2}(Q)$, where the operator $\mathcal{H}_{T}$ acts only on the time variable $t$. The stability estimate (3.6) implies the stability estimate

$$
\frac{1}{2}\|u\|_{H_{0 ; 0,}^{1,1 / 2}(Q)} \leq \sup _{0 \neq v \in H_{0 ; 0,}^{1,1 / 2}(Q)} \frac{\left\langle\partial_{t} u, \mathcal{H}_{T} v\right\rangle_{Q}+\left\langle\nabla_{x} u, \nabla_{x} \mathcal{H}_{T} v\right\rangle_{L^{2}(Q)}}{\|v\|_{H_{0 ; 0,}^{1,1 / 2}(Q)}}
$$




\section{ETNA}

Kent State University and

Johann Radon Institute (RICAM)

for all $u \in H_{0 ; 0}^{1,1 / 2}(Q)$, and therefore unique solvability of the variational formulation (3.7) follows.

When using some conforming space-time finite element space $\mathcal{V}_{h}=\operatorname{span}\left\{\phi_{k}\right\}_{k=1}^{M} \subset$ $H_{0 ; 0}^{1,1 / 2}(Q)$, the Galerkin variational formulation of (3.7) is to find $u_{h} \in \mathcal{V}_{h}$ such that

$$
\left\langle\partial_{t} u_{h}, \mathcal{H}_{T} v_{h}\right\rangle_{L^{2}(Q)}+\left\langle\nabla_{x} u_{h}, \nabla_{x} \mathcal{H}_{T} v_{h}\right\rangle_{L^{2}(Q)}=\left\langle f, \mathcal{H}_{T} v_{h}\right\rangle_{Q}
$$

is satisfied for all $v_{h} \in \mathcal{V}_{h}$, which is equivalent to the linear system of algebraic equations, $K_{h} \underline{u}=\underline{f}$. The stiffness matrix is given as $K_{h}=A_{h}+B_{h}$ with

$$
A_{h}[\ell, k]=\left\langle\partial_{t} \phi_{k}, \mathcal{H}_{T} \phi_{\ell}\right\rangle_{L^{2}(Q)}, \quad B_{h}[\ell, k]=\left\langle\nabla_{x} \phi_{k}, \nabla_{x} \mathcal{H}_{T} \phi_{\ell}\right\rangle_{L^{2}(Q)}, \quad k, \ell=1, \ldots, M .
$$

Note that $A_{h}$ is symmetric and positive definite, while in general, $B_{h}$ is not symmetric but positive definite and ill-conditioned. Hence, $K_{h}$ is positive definite, and unique solvability of (3.8) follows for any conforming choice of the space-time basis functions $\phi_{k}$. However, to perform the temporal transformation $\mathcal{H}_{T}$ easily and to be able to present an a priori error analysis, here we will consider a space-time tensor-product finite element space only.

Let $W_{h_{x}}=\operatorname{span}\left\{\psi_{i}\right\}_{i=1}^{M_{x}} \subset H_{0}^{1}(\Omega)$ be some spatial finite element space, e.g., of piecewise linear or bilinear continuous basis functions $\psi_{i}$, which are defined with respect to some admissible and globally quasi-uniform finite element mesh with mesh size $h_{x}$. As before, $V_{h_{t}}=S_{h_{t}}^{1}(0, T) \cap H_{0}^{1 / 2}(0, T)=\operatorname{span}\left\{\varphi_{k}\right\}_{k=1}^{N_{t}}$ is the space of piecewise linear functions, which are defined with respect to some globally quasi-uniform finite element mesh with mesh size $h_{t}$. Hence, we introduce the tensor-product space-time finite element space $\mathcal{V}_{h}:=W_{h_{x}} \otimes V_{h_{t}}$.

For a given $v \in H_{0}^{1 / 2}\left(0, T ; L^{2}(\Omega)\right)$, we define the $H_{0}^{1 / 2}$-projection $Q_{h_{t}}^{1 / 2} v \in L^{2}(\Omega) \otimes V_{h_{t}}$ as the unique solution of the variational problem

$$
\left\langle\partial_{t} Q_{h_{t}}^{1 / 2} v, \mathcal{H}_{T} v_{h_{t}}\right\rangle_{L^{2}(Q)}=\left\langle\partial_{t} v, \mathcal{H}_{T} v_{h_{t}}\right\rangle_{Q}
$$

for all $v_{h_{t}} \in L^{2}(\Omega) \otimes V_{h_{t}}$. Moreover, for $v \in L^{2}\left(0, T ; H_{0}^{1}(\Omega)\right)$, we define the $H_{0}^{1}$-projection $Q_{h_{x}}^{1} v \in W_{h_{x}} \otimes L^{2}(0, T)$ as the unique solution of the variational problem

$$
\int_{0}^{T} \int_{\Omega} \nabla_{x} Q_{h_{x}}^{1} v(x, t) \cdot \nabla_{x} v_{h_{x}}(x, t) d x d t=\int_{0}^{T} \int_{\Omega} \nabla_{x} v(x, t) \cdot \nabla_{x} v_{h_{x}}(x, t) d x d t
$$

for all $v_{h_{x}} \in W_{h_{x}} \otimes L^{2}(0, T)$. It turns out that $Q_{h_{t}}^{1 / 2} Q_{h_{x}}^{1} v \in \mathcal{V}_{h}$ is well-defined when assuming $\partial_{t} v \in L^{2}\left(0, T ; H_{0}^{1}(\Omega)\right)$ and $\nabla_{x} v \in H_{0}^{1 / 2}\left(0, T ; L^{2}(\Omega)\right)$, respectively, and that the projection operators $Q_{h_{t}}^{1 / 2}, Q_{h_{x}}^{1}$ and partial derivatives $\partial_{t}, \nabla_{x}$ commute in space and time [45].

THEOREM 3.4. Let $u \in H_{0 ; 0}^{1,1 / 2}(Q)$ and $u_{h} \in \mathcal{V}_{h}$ be the unique solutions of the variational problems (3.7) and (3.8), respectively. If $u$ is sufficiently regular and the spatial domain $\Omega$ is assumed to be either convex or has a smooth boundary $\Gamma$, then there hold true the error estimates

$$
\begin{aligned}
\left\|u-u_{h}\right\|_{H_{0,}^{1 / 2}\left(0, T ; L^{2}(\Omega)\right)} \leq & c_{1} h_{t}^{3 / 2}\|u\|_{H^{2}\left(0, T ; L^{2}(\Omega)\right)}+c_{2} h_{x}^{3 / 2}\|u\|_{H_{0}^{1 / 2}\left(0, T ; H^{3 / 2}(\Omega)\right)} \\
& +c_{3} h_{t}^{1 / 2} h_{x}\left\|\partial_{t} \nabla_{x} u\right\|_{L^{2}(Q)}+c_{4} h_{t}^{3 / 2}\left\|\partial_{t} \Delta_{x} u\right\|_{L^{2}(Q)}
\end{aligned}
$$

and

$$
\left\|u-u_{h}\right\|_{L^{2}(Q)} \leq c_{1} h_{t}^{2}\|u\|_{H^{2}\left(0, T ; L^{2}(\Omega)\right)}+c_{2} h_{x}^{2}\|u\|_{L^{2}\left(0, T ; H^{2}(\Omega)\right)}+c_{3} h_{t} h_{x}\left\|\partial_{t} \nabla_{x} u\right\|_{L^{2}(Q)}
$$

$$
+c_{4} h_{x}^{2}\left\|\partial_{t} u\right\|_{L^{2}\left(0, T ; H^{2}(\Omega)\right)}+c_{5} h_{t}^{2}\left\|\Delta_{x} u\right\|_{H^{2}\left(0, T ; L^{2}(\Omega)\right)} .
$$


Proof. With the norm representation in $H_{0}^{1 / 2}\left(0, T ; L^{2}(\Omega)\right)$, the positivity (2.10), and the Galerkin orthogonality of the variational formulations (3.7) and (3.8), we have for $v_{h}=Q_{h_{t}}^{1 / 2} Q_{h_{x}}^{1} u \in \mathcal{V}_{h}$, using the definitions of the projections $Q_{h_{t}}^{1 / 2}$ and $Q_{h_{x}}^{1}$ and integration by parts spatially,

$$
\begin{aligned}
& \left\|u_{h}-Q_{h_{t}}^{1 / 2} Q_{h_{x}}^{1} u\right\|_{H_{0}^{1 / 2}\left(0, T ; L^{2}(\Omega)\right)}^{2}=\left\langle\partial_{t}\left(u_{h}-Q_{h_{t}}^{1 / 2} Q_{h_{x}}^{1} u\right), \mathcal{H}_{T}\left(u_{h}-Q_{h_{t}}^{1 / 2} Q_{h_{x}}^{1} u\right)\right\rangle_{Q} \\
& \leq\left\langle\partial_{t}\left(u_{h}-Q_{h_{t}}^{1 / 2} Q_{h_{x}}^{1} u\right), \mathcal{H}_{T}\left(u_{h}-Q_{h_{t}}^{1 / 2} Q_{h_{x}}^{1} u\right)\right\rangle_{Q} \\
& +\left\langle\nabla_{x}\left(u_{h}-Q_{h_{t}}^{1 / 2} Q_{h_{x}}^{1} u\right), \nabla_{x} \mathcal{H}_{T}\left(u_{h}-Q_{h_{t}}^{1 / 2} Q_{h_{x}}^{1} u\right)\right\rangle_{L^{2}(Q)} \\
& =\left\langle\partial_{t}\left(u-Q_{h_{t}}^{1 / 2} Q_{h_{x}}^{1} u\right), \mathcal{H}_{T}\left(u_{h}-Q_{h_{t}}^{1 / 2} Q_{h_{x}}^{1} u\right)\right\rangle_{Q} \\
& +\left\langle\nabla_{x}\left(u-Q_{h_{t}}^{1 / 2} Q_{h_{x}}^{1} u\right), \nabla_{x} \mathcal{H}_{T}\left(u_{h}-Q_{h_{t}}^{1 / 2} Q_{h_{x}}^{1} u\right)\right\rangle_{L^{2}(Q)} \\
& =\left\langle\partial_{t}\left(u-Q_{h_{x}}^{1} u\right), \mathcal{H}_{T}\left(u_{h}-Q_{h_{t}}^{1 / 2} Q_{h_{x}}^{1} u\right)\right\rangle_{Q} \\
& +\left\langle\nabla_{x}\left(u-Q_{h_{t}}^{1 / 2} u\right), \nabla_{x} \mathcal{H}_{T}\left(u_{h}-Q_{h_{t}}^{1 / 2} Q_{h_{x}}^{1} u\right)\right\rangle_{L^{2}(Q)} \\
& \text { (3.11) }=\left\langle\partial_{t}\left(u-Q_{h_{x}}^{1} u\right), \mathcal{H}_{T}\left(u_{h}-Q_{h_{t}}^{1 / 2} Q_{h_{x}}^{1} u\right)\right\rangle_{Q} \\
& -\left\langle\Delta_{x}\left(u-Q_{h_{t}}^{1 / 2} u\right), \mathcal{H}_{T}\left(u_{h}-Q_{h_{t}}^{1 / 2} Q_{h_{x}}^{1} u\right)\right\rangle_{Q} \\
& \leq\left\|u-Q_{h_{x}}^{1} u\right\|_{H_{0,}^{1 / 2}\left(0, T ; L^{2}(\Omega)\right)}\left\|u_{h}-Q_{h_{t}}^{1 / 2} Q_{h_{x}}^{1} u\right\|_{H_{0,}^{1 / 2}\left(0, T ; L^{2}(\Omega)\right)} \\
& +\left\|\Delta_{x}\left(u-Q_{h_{t}}^{1 / 2} u\right)\right\|_{\left[H_{, 0}^{1 / 2}\left(0, T ; L^{2}(\Omega)\right)\right]^{\prime}}\left\|u_{h}-Q_{h_{t}}^{1 / 2} Q_{h_{x}}^{1} u\right\|_{H_{0}^{1 / 2}\left(0, T ; L^{2}(\Omega)\right)},
\end{aligned}
$$

i.e.,

$$
\begin{aligned}
& \left\|u_{h}-Q_{h_{t}}^{1 / 2} Q_{h_{x}}^{1} u\right\|_{H_{0,}^{1 / 2}\left(0, T ; L^{2}(\Omega)\right)} \\
& \quad \leq\left\|u-Q_{h_{x}}^{1} u\right\|_{H_{0,}^{1 / 2}\left(0, T ; L^{2}(\Omega)\right)}+\left\|\Delta_{x}\left(u-Q_{h_{t}}^{1 / 2} u\right)\right\|_{\left[H_{, 0}^{1 / 2}\left(0, T ; L^{2}(\Omega)\right)\right]^{\prime}} .
\end{aligned}
$$

Hence, we have

$$
\begin{aligned}
\| u- & u_{h} \|_{H_{0}^{1 / 2}\left(0, T ; L^{2}(\Omega)\right)} \\
\leq \| u & -Q_{h_{t}}^{1 / 2} Q_{h_{x}}^{1} u\left\|_{H_{0,}^{1 / 2}\left(0, T ; L^{2}(\Omega)\right)}+\right\| u_{h}-Q_{h_{t}}^{1 / 2} Q_{h_{x}}^{1} u \|_{H_{0,}^{1 / 2}\left(0, T ; L^{2}(\Omega)\right)} \\
\leq \| u & -Q_{h_{t}}^{1 / 2} Q_{h_{x}}^{1} u \|_{H_{0,}^{1 / 2}\left(0, T ; L^{2}(\Omega)\right)} \\
& +\left\|u-Q_{h_{x}}^{1} u\right\|_{H_{0,}^{1 / 2}\left(0, T ; L^{2}(\Omega)\right)}+\left\|\Delta_{x}\left(u-Q_{h_{t}}^{1 / 2} u\right)\right\|_{\left[H_{, 0}^{1 / 2}\left(0, T ; L^{2}(\Omega)\right)\right]^{\prime}} \\
\leq \| u- & Q_{h_{t}}^{1 / 2} u\left\|_{H_{0}^{1 / 2}\left(0, T ; L^{2}(\Omega)\right)}+\right\| u-Q_{h_{x}}^{1} u \|_{H_{0}^{1 / 2}\left(0, T ; L^{2}(\Omega)\right)} \\
& +\left\|\left(I-Q_{h_{t} / 2}^{1 / 2}\right)\left(u-Q_{h_{x}}^{1} u\right)\right\|_{H_{0}^{1 / 2}\left(0, T ; L^{2}(\Omega)\right)} \\
& +\left\|u-Q_{h_{x}}^{1} u\right\|_{H_{0,}^{1 / 2}\left(0, T ; L^{2}(\Omega)\right)}+\left\|\Delta_{x}\left(u-Q_{h_{t}}^{1 / 2} u\right)\right\|_{\left[H_{, 0}^{1 / 2}\left(0, T ; L^{2}(\Omega)\right)\right]^{\prime}},
\end{aligned}
$$

and the energy error estimate (3.9) follows from standard error estimates for the involved projection operators. 
With a Poincaré-Friedrichs-type inequality and relation (3.11), we also have

$$
\begin{aligned}
\frac{1}{c}\left\|u_{h}-Q_{h_{t}}^{1 / 2} Q_{h_{x}}^{1} u\right\|_{L^{2}(Q)}^{2} \leq & \left\|u_{h}-Q_{h_{t}}^{1 / 2} Q_{h_{x}}^{1} u\right\|_{H_{0}^{1 / 2}\left(0, T ; L^{2}(\Omega)\right)}^{2} \\
\leq & \left\langle\partial_{t}\left(u-Q_{h_{x}}^{1} u\right), \mathcal{H}_{T}\left(u_{h}-Q_{h_{t}}^{1 / 2} Q_{h_{x}}^{1} u\right)\right\rangle_{Q} \\
& \quad-\left\langle\Delta_{x}\left(u-Q_{h_{t}}^{1 / 2} u\right), \mathcal{H}_{T}\left(u_{h}-Q_{h_{t}}^{1 / 2} Q_{h_{x}}^{1} u\right)\right\rangle_{L^{2}(Q)} \\
\leq\left\|\partial_{t}\left(u-Q_{h_{x}}^{1} u\right)\right\|_{L^{2}(Q)}\left\|u_{h}-Q_{h_{t}}^{1 / 2} Q_{h_{x}}^{1} u\right\|_{L^{2}(Q)} & \quad+\left\|\Delta_{x}\left(u-Q_{h_{t}}^{1 / 2} u\right)\right\|_{L^{2}(Q)}\left\|u_{h}-Q_{h_{t}}^{1 / 2} Q_{h_{x}}^{1} u\right\|_{L^{2}(Q)},
\end{aligned}
$$

which implies

$$
\left\|u_{h}-Q_{h_{t}}^{1 / 2} Q_{h_{x}}^{1} u\right\|_{L^{2}(Q)} \leq c\left\|\partial_{t}\left(u-Q_{h_{x}}^{1} u\right)\right\|_{L^{2}(Q)}+c\left\|\Delta_{x}\left(u-Q_{h_{t}}^{1 / 2} u\right)\right\|_{L^{2}(Q)} .
$$

Therefore

$$
\begin{gathered}
\left\|u-u_{h}\right\|_{L^{2}(Q)} \leq\left\|u-Q_{h_{t}}^{1 / 2} Q_{h_{x}}^{1} u\right\|_{L^{2}(Q)}+\left\|u_{h}-Q_{h_{t}}^{1 / 2} Q_{h_{x}}^{1} u\right\|_{L^{2}(Q)} \\
\leq\left\|u-Q_{h_{t}}^{1 / 2} Q_{h_{x}}^{1} u\right\|_{L^{2}(Q)}+c\left\|\partial_{t}\left(u-Q_{h_{x}}^{1} u\right)\right\|_{L^{2}(Q)}+c \| \Delta_{x}\left(u-Q_{h_{t}}^{1 / 2} u \|_{L^{2}(Q)}\right. \\
\leq\left\|u-Q_{h_{t}}^{1 / 2} u\right\|_{L^{2}(Q)}+\left\|u-Q_{h_{x}}^{1} u\right\|_{L^{2}(Q)}+\left\|\left(I-Q_{h_{t}}^{1 / 2}\right)\left(u-Q_{h_{x}}^{1} u\right)\right\|_{L^{2}(Q)} \\
+c\left\|\partial_{t}\left(u-Q_{h_{x}}^{1} u\right)\right\|_{L^{2}(Q)}+c\left\|\Delta_{x}\left(u-Q_{h_{t}}^{1 / 2} u\right)\right\|_{L^{2}(Q)} .
\end{gathered}
$$

Finally, (3.10) follows again from standard error estimates for the projection operators.

As a numerical example, we consider the solution $u(x, t)=\sin \left(\frac{5 \pi}{4} t\right) \sin (\pi x)$ for $(x, t) \in Q$ with $Q:=(0,1) \times(0,2)$. For a uniform discretisation of the Galerkin variational formulation (3.8) with the tensor-product space-time finite element space $\mathcal{V}_{h}=W_{h_{x}} \otimes V_{h_{t}}$, we use the mesh sizes $h_{x}=1 / M_{x}$ and $h_{t}=2 / N_{t}$ with $M_{x}=N_{t}=2^{j}, j=1, \ldots, 8$. Since the solution $u$ is smooth, we expect second-order convergence in $L^{2}(Q)$ (see (3.10)) and first-order convergence in $H^{1}(Q)$. Note that the latter follows by standard arguments when using the $H^{1}(Q)$-projection and an inverse inequality. The predicted convergence orders are confirmed by the numerical results given in Table 3.1. There, we also present numerical results for the spectral condition number of the discretised system, which behaves asymptotically as $h_{x}^{-2}$, as expected.

TABLE 3.1

Convergence rates of the Galerkin-Bubnov formulation (3.8).

\begin{tabular}{rrccccccr}
$M_{x}, N_{t}$ & dof & $h_{x}$ & $h_{t}$ & $\left\|u-u_{h}\right\|_{L^{2}}$ & eoc & $\left|u-u_{h}\right|_{H^{1}}$ & eoc & $\kappa_{2}\left(K_{h}\right)$ \\
\hline 2 & 2 & 0.5000 & 1.0000 & 0.9108053 & - & 4.48437 & - & 2.5 \\
4 & 12 & 0.2500 & 0.5000 & 0.1577439 & 2.5 & 1.89083 & 1.2 & 19.8 \\
8 & 56 & 0.1250 & 0.2500 & 0.0293609 & 2.4 & 0.84239 & 1.2 & 63.3 \\
16 & 240 & 0.0625 & 0.1250 & 0.0068950 & 2.1 & 0.41496 & 1.0 & 170.9 \\
32 & 992 & 0.0312 & 0.0625 & 0.0016957 & 2.0 & 0.20679 & 1.0 & 484.0 \\
64 & 4032 & 0.0156 & 0.0312 & 0.0004220 & 2.0 & 0.10331 & 1.0 & 1691.4 \\
128 & 16256 & 0.0078 & 0.0156 & 0.0001054 & 2.0 & 0.05165 & 1.0 & 6623.3 \\
256 & 65280 & 0.0039 & 0.0078 & 0.0000263 & 2.0 & 0.02582 & 1.0 & 26355.9
\end{tabular}


REMARK 3.5. Numerical results [45] indicate that the stability constant $c_{S}$ of the discrete inf-sup condition

$$
c_{S}\left\|u_{h}\right\|_{H_{0 ; 0}^{1,1 / 2}(Q)} \leq \sup _{0 \neq v_{h} \in \mathcal{V}_{h}} \frac{\left\langle\partial_{t} u_{h}, \mathcal{H}_{T} v_{h}\right\rangle_{L^{2}(Q)}+\left\langle\nabla_{x} u_{h}, \nabla_{x} \mathcal{H}_{T} v_{h}\right\rangle_{L^{2}(Q)}}{\left\|v_{h}\right\|_{H_{0 ; 0}^{1,1 / 2}(Q)}}
$$

for all $u_{h} \in \mathcal{V}_{h}$ is mesh dependent, i.e., $c_{S}=\mathcal{O}\left(\max \left\{h_{t}, h_{x}\right\}\right)$. However, it seems to be possible to derive almost optimal energy error estimates also in this case. Since this is far beyond the scope of this paper, this will be discussed elsewhere.

REMARK 3.6. The use of tensor-product approximations allows the implementation of the transformation $\mathcal{H}_{T}$ by the series representation (2.8). Based on the kernel representation (2.11), one can derive alternative representations [39] for the bilinear forms including $\mathcal{H}_{T}$, e.g.,

$$
\begin{aligned}
\int_{0}^{T} \int_{\Omega} \partial_{t} u(x, t)\left(\mathcal{H}_{T} v\right)(x, t) d x d t \\
\quad=-\frac{1}{\pi} \int_{\Omega} \int_{0}^{T} \partial_{t} u(x, t) \int_{0}^{T} \ln \left[\tan \frac{\pi(s+t)}{4 T} \tan \frac{\pi|t-s|}{4 T}\right] \partial_{t} v(x, s) d s d t d x
\end{aligned}
$$

which admits not only the use of hierarchical matrices for acceleration but can also be used for more general space-time finite element meshes.

4. Second-order ordinary differential equations. As in (2.1), we consider the initial value problem

$$
\partial_{t t} u(t)=f(t) \quad \text { for } t \in(0, T), \quad u(0)=\partial_{t} u(0)=0 .
$$

When multiplying the differential equation with a test function $w$ satisfying $w(T)=0$, integrating over $(0, T)$, and applying integration by parts once, this results in the variational formulation to find $u \in H_{0}^{1},(0, T)$ such that

$$
-\int_{0}^{T} \partial_{t} u(t) \partial_{t} w(t) d t=\langle f, w\rangle_{(0, T)}
$$

is satisfied for all $w \in H_{, 0}^{1}(0, T)$, where $f \in\left[H_{, 0}^{1}(0, T)\right]^{\prime}$ is given. Note that the initial condition $u(0)=0$ is considered in the strong sense, whereas the initial condition $\partial_{t} u(0)=0$ is incorporated in the variational formulation. The bilinear form

$$
a(u, w):=-\int_{0}^{T} \partial_{t} u(t) \partial_{t} w(t) d t \quad \text { for } u \in H_{0}^{1}(0, T), w \in H_{, 0}^{1}(0, T)
$$

is obviously bounded, and therefore it remains to establish some stability or ellipticity estimate to ensure unique solvability of the variational formulation (4.2). For this, we use the concept of an optimal test function; see Remark 2.2. It turns out that for $u \in H_{0,}^{1}(0, T)$, we can define the transformation $\overline{\mathcal{H}}_{T} u \in H_{, 0}^{1}(0, T)$,

$$
\left(\overline{\mathcal{H}}_{T} u\right)(t):=u(T)-u(t), \quad t \in(0, T) .
$$

The operator $\overline{\mathcal{H}}_{T}$, as defined in (4.3), is obviously norm preserving satisfying

$$
\left\|\overline{\mathcal{H}}_{T} u\right\|_{H_{, 0}^{1}(0, T)}=\|u\|_{H_{0,}^{1}(0, T)} \quad \text { for all } u \in H_{0,}^{1}(0, T),
$$


and for $u, v \in H_{0}^{1},(0, T)$, there holds true the symmetry relation

$$
a\left(u, \overline{\mathcal{H}}_{T} v\right)=a\left(v, \overline{\mathcal{H}}_{T} u\right)=\int_{0}^{T} \partial_{t} u(t) \partial_{t} v(t) d t
$$

which also implies ellipticity,

$$
a\left(u, \overline{\mathcal{H}}_{T} u\right)=\left\|\partial_{t} u\right\|_{L^{2}(0, T)}^{2} \quad \text { for all } u \in H_{0,}^{1}(0, T) .
$$

However, the form

$$
\left\langle u, \overline{\mathcal{H}}_{T} u\right\rangle_{L^{2}(0, T)}=\int_{0}^{T} u(t)[u(T)-u(t)] d t
$$

is indefinite, i.e., a result as (2.10) for the transformation $\mathcal{H}_{T}$ does not hold true for $\overline{\mathcal{H}}_{T}$.

For a finite element discretisation of the variational formulation (4.2), we use the same notations as in Section 2. In particular, we have to find $u_{h} \in V_{h}:=S_{h}^{1}(0, T) \cap H_{0}^{1}(0, T)$ such that

$$
-\left\langle\partial_{t} u_{h}, \partial_{t} \overline{\mathcal{H}}_{T} v_{h}\right\rangle_{L^{2}(0, T)}=\left\langle f, \overline{\mathcal{H}}_{T} v_{h}\right\rangle_{(0, T)} \quad \text { for all } v_{h} \in V_{h}
$$

As before, we have unique solvability of (4.4), and the a priori error estimate (2.15) remains valid, where for $\sigma=1$, this corresponds to the energy error estimate, while for $\sigma=0$, we have to apply a Nitsche-type argument.

For the numerical example, we consider the solution $u(t)=\sin ^{2}\left(\frac{5}{4} \pi t\right)$, for $t \in(0, T)$, with $T=2$. The numerical results are given in Table 4.1, where we observe optimal order of convergence as predicted.

TABLE 4.1

Numerical results for the Galerkin-Bubnov formulation (4.4).

\begin{tabular}{rcccccrrr}
$N$ & $\left\|u-u_{h}\right\|_{L^{2}}$ & eoc & $\left\|\partial_{t}\left(u-u_{h}\right)\right\|_{L^{2}}$ & eoc & $\lambda_{\min }\left(K_{h}\right)$ & $\lambda_{\max }\left(K_{h}\right)$ & $\kappa_{2}\left(K_{h}\right)$ \\
\hline 4 & 0.49700 & - & 3.4650 & - & 0.2412 & 7.1 & 29 \\
8 & 0.16170 & 1.6 & 2.0880 & 0.7 & 0.1362 & 15.5 & 114 \\
16 & 0.04307 & 1.9 & 1.0950 & 0.9 & 0.0724 & 31.7 & 438 \\
32 & 0.01094 & 2.0 & 0.5542 & 1.0 & 0.0374 & 63.9 & 1709 \\
64 & 0.00275 & 2.0 & 0.2780 & 1.0 & 0.0189 & 127.9 & 6741 \\
128 & 0.00069 & 2.0 & 0.1391 & 1.0 & 0.0095 & 256.0 & 26765 \\
256 & 0.00017 & 2.0 & 0.0696 & 1.0 & 0.0048 & 512.0 & 106655
\end{tabular}

The stiffness matrix of the Galerkin-Bubnov finite element formulation (4.4) is symmetric and positive definite, and its spectral behaviour is as known for finite element discretisations of second-order partial differential equations. Moreover, due to (4.3), we have for the piecewise linear basis functions $\varphi_{k} \in H_{0}^{1}(0, T), k=1, \ldots, N$,

$$
\left(\overline{\mathcal{H}}_{T} \varphi_{k}\right)(t)=-\varphi_{k}(t) \quad \text { for } k=1, \ldots, N-1,
$$

and

$$
\left(\overline{\mathcal{H}}_{T} \varphi_{N}\right)(t)=\left\{\begin{array}{cl}
1 & \text { for } t \in\left[0, t_{N-1}\right], \\
\frac{T-t}{T-t_{N-1}} & \text { for } t \in\left(t_{N-1}, T\right]
\end{array}\right.
$$


and hence

$$
\overline{\mathcal{H}}_{T} V_{h}=\operatorname{span}\left\{\varphi_{k}\right\}_{k=0}^{N-1}
$$

Instead of (4.1), for $\mu=\nu^{2}>0$, we consider the second-order ordinary differential equation

$$
\partial_{t t} u(t)+\mu u(t)=f(t) \quad \text { for } t \in(0, T), \quad u(0)=\partial_{t} u(0)=0,
$$

and the variational formulation to find $u \in H_{0,}^{1}(0, T)$ such that

$$
a\left(u, \overline{\mathcal{H}}_{T} v\right):=-\int_{0}^{T} \partial_{t} u(t) \partial_{t}\left(\overline{\mathcal{H}}_{T} v\right)(t) d t+\mu \int_{0}^{T} u(t)\left(\overline{\mathcal{H}}_{T} v\right)(t) d t=\left\langle f, \overline{\mathcal{H}}_{T} v\right\rangle_{(0, T)}
$$

is satisfied for all $v \in H_{0}^{1}(0, T)$, where $f \in\left[H_{, 0}^{1}(0, T)\right]^{\prime}$ is given.

THEOREM 4.1. For given $f \in\left[H_{, 0}^{1}(0, T)\right]^{\prime}$, the variational formulation (4.7) admits a unique solution $u \in H_{0,}^{1}(0, T)$ satisfying

$$
\|u\|_{H_{0,(0, T)}^{1}} \leq c\|f\|_{\left[H_{, 0}^{1}(0, T)\right]^{\prime}} .
$$

Proof. By using the Riesz representation theorem, we rewrite the variational problem (4.7) as an operator equation

$$
\mathcal{A} u+\mu \mathcal{C} u=\bar{f}
$$

where $\mathcal{A}: H_{0}^{1}(0, T) \rightarrow\left[H_{0}^{1}(0, T)\right]^{\prime}$, defined via

$$
\langle\mathcal{A} u, v\rangle=-\left\langle\partial_{t} u, \partial_{t} \overline{\mathcal{H}}_{T} v\right\rangle_{L^{2}(0, T)} \quad \text { for } u, v \in H_{0,}^{1}(0, T),
$$

is elliptic, and hence invertible, and $\mathcal{C}: H_{0,}^{1}(0, T) \rightarrow\left[H_{0,}^{1}(0, T)\right]^{\prime}$, defined via

$$
\langle\mathcal{C} u, v\rangle=\left\langle u, \overline{\mathcal{H}}_{T} v\right\rangle_{L^{2}(0, T)} \text { for } u, v \in H_{0,}^{1}(0, T),
$$

is compact. Hence, we can apply the Fredholm alternative, and it remains to ensure the injectivity of $\mathcal{A}+\mu \mathcal{C}$. Let $u \in H_{0}^{1},(0, T)$ be a solution of the homogeneous equation $(\mathcal{A}+\mu \mathcal{C}) u=0$, i.e.,

$$
\left\langle\partial_{t} u, \partial_{t} w\right\rangle_{L^{2}(0, T)}=\mu\langle u, w\rangle_{L^{2}(0, T)} \quad \text { for all } w \in H_{, 0}^{1}(0, T)
$$

This is the weak formulation of the eigenvalue problem

$$
-\partial_{t t} u(t)=\mu u(t) \quad \text { for } t \in(0, T), \quad u(0)=\partial_{t} u(0)=0,
$$

which only admits the trivial solution $u \equiv 0$.

While the result of Theorem 4.1 ensures unique solvability of the variational formulation (4.7), it does not include an explicit dependence on the parameter $\mu$. Hence, we will provide a stability estimate from which we can conclude such a result.

LEMMA 4.2. For $u \in H_{0,}^{1}(0, T)$ there holds true the stability estimate

$$
\frac{2}{2+\nu T}\left\|\partial_{t} u\right\|_{L^{2}(0, T)} \leq \sup _{0 \neq v \in H_{, 0}^{1}(0, T)} \frac{a(u, v)}{\left\|\partial_{t} v\right\|_{L^{2}(0, T)}}
$$


Proof. For given $u \in H_{0,}^{1}(0, T)$ and suitable chosen $w \in H_{, 0}^{2}(0, T)$, we consider the test function $v:=\overline{\mathcal{H}}_{T} u+w \in H_{, 0}^{1}(0, T)$. Then,

$$
\begin{aligned}
a(u, v)= & -\int_{0}^{T} \partial_{t} u(t) \partial_{t}[-u(t)+w(t)] d t+\mu \int_{0}^{T} u(t)[u(T)-u(t)+w(t)] d t \\
= & \int_{0}^{T}\left[\partial_{t} u(t)\right]^{2} d t-\int_{0}^{T} \partial_{t} u(t) \partial_{t} w(t) d t+\mu \int_{0}^{T} u(t)[u(T)-u(t)+w(t)] d t \\
= & \int_{0}^{T}\left[\partial_{t} u(t)\right]^{2} d t-\left.u(t) \partial_{t} w(t)\right|_{0} ^{T}+\int_{0}^{T} u(t) \partial_{t t} w(t) d t \\
& \quad+\mu \int_{0}^{T} u(t)[u(T)-u(t)+w(t)] d t \\
= & \int_{0}^{T}\left[\partial_{t} u(t)\right]^{2} d t-u(T) \partial_{t} w(T) \\
= & \quad+\int_{0}^{T} u(t)\left[\partial_{t t} w(t)+\mu(u(T)-u(t)+w(t))\right] d t
\end{aligned}
$$

if

$$
\partial_{t t} w(t)+\mu w(t)=\mu[u(t)-u(T)] \quad \text { for } t \in(0, T), \quad w(T)=\partial_{t} w(T)=0
$$

is satisfied. Using $\mu=\nu^{2}$, we obtain

$$
w(t)=\nu \int_{t}^{T} \sin (\nu(s-t))[u(s)-u(T)] d s,
$$

and therefore,

$$
\begin{aligned}
\partial_{t} w(t) & =-\nu^{2} \int_{t}^{T} \cos (\nu(s-t))[u(s)-u(T)] d s \\
& =-\left.\nu \sin (\nu(s-t))[u(t)-u(T)]\right|_{t} ^{T}+\nu \int_{t}^{T} \sin (\nu(s-t)) \partial_{s} u(s) d s \\
& =\nu \int_{t}^{T} \sin (\nu(s-t)) \partial_{s} u(s) d s
\end{aligned}
$$

follows. Further, with

$$
\begin{aligned}
{\left[\partial_{t} w(t)\right]^{2} } & =\nu^{2}\left[\int_{t}^{T} \sin (\nu(s-t)) \partial_{s} u(s) d s\right]^{2} \\
& \leq \nu^{2} \int_{t}^{T} \sin ^{2}(\nu(s-t)) d s \int_{t}^{T}\left[\partial_{s} u(s)\right]^{2} d s \\
& \leq \nu^{2} \int_{t}^{T} \sin ^{2}(\nu(s-t)) d s \int_{0}^{T}\left[\partial_{t} u(t)\right]^{2} d t
\end{aligned}
$$


we conclude that

$$
\begin{aligned}
\int_{0}^{T}\left[\partial_{t} w(t)\right]^{2} d t & \leq \nu^{2} \int_{0}^{T} \int_{t}^{T} \sin ^{2}(\nu(s-t)) d s d t \int_{0}^{T}\left[\partial_{t} u(t)\right]^{2} d t \\
& =\nu^{2} \frac{1}{4} \frac{\cos ^{2}(\nu T)-1+\nu^{2} T^{2}}{\nu^{2}} \int_{0}^{T}\left[\partial_{t} u(t)\right]^{2} d t \\
& \leq \frac{1}{4} \nu^{2} T^{2} \int_{0}^{T}\left[\partial_{t} u(t)\right]^{2} d t
\end{aligned}
$$

i.e.,

$$
\left\|\partial_{t} w\right\|_{L^{2}(0, T)} \leq \frac{1}{2} \nu T\left\|\partial_{t} u\right\|_{L^{2}(0, T)} .
$$

Finally, with this, we have

$$
\begin{aligned}
\left\|\partial_{t} v\right\|_{L^{2}(0, T)} & =\left\|\partial_{t} w-\partial_{t} u\right\|_{L^{2}(0, T)} \\
& \leq\left\|\partial_{t} w\right\|_{L^{2}(0, T)}+\left\|\partial_{t} u\right\|_{L^{2}(0, T)} \leq\left(1+\frac{1}{2} \nu T\right)\left\|\partial_{t} u\right\|_{L^{2}(0, T)},
\end{aligned}
$$

and therefore,

$$
a(u, v)=\left\|\partial_{t} u\right\|_{L^{2}(0, T)}^{2} \geq \frac{2}{2+\nu T}\left\|\partial_{t} u\right\|_{L^{2}(0, T)}\left\|\partial_{t} v\right\|_{L^{2}(0, T)}
$$

follows, which implies the stability condition as stated.

While Theorem 4.1 implies unique solvability of the variational formulation (4.7), we can use the stability condition (4.8) to obtain a bound for the solution $u$, which explicitely depends on $\nu$.

COROLLARY 4.3. For the unique solution $u \in H_{0,}^{1}(0, T)$ of the variational formulation (4.7), there holds true that

$$
\left\|\partial_{t} u\right\|_{L^{2}(0, T)} \leq\left(1+\frac{1}{2} \nu T\right)\|f\|_{\left[H_{, 0}^{1}(0, T)\right]^{\prime}} .
$$

REMARK 4.4. We consider the initial value problem (4.6) for $f(t)=\sin (\nu t)$ with the solution

$$
u(t)=\frac{1}{2 \nu^{2}}[\sin (\nu t)-\nu t \cos (\nu t)], \quad \partial_{t} u(t)=\frac{1}{2} t \sin (\nu t) .
$$

For this, we compute

$$
\begin{aligned}
\left\|\partial_{t} u\right\|_{L^{2}(0, T)}^{2} & =\frac{1}{48} \frac{1}{\nu^{3}}\left[2 \nu^{3} T^{3}+3 \nu T-6 \nu^{2} T^{2} \cos (\nu T) \sin (\nu T)\right. \\
& \left.\quad-6 \nu T \cos ^{2}(\nu T)+3 \cos (\nu T) \sin (\nu T)\right] \\
\simeq & \\
&
\end{aligned}
$$

as $\nu \rightarrow \infty$. On the other hand, we determine $w \in H_{, 0}^{1}(0, T)$ as unique solution of the boundary value problem

$$
-\partial_{t t} w(t)=f(t) \quad \text { for } t \in(0, T), \quad \partial_{t} w(0)=w(T)=0,
$$


i.e.,

$$
\partial_{t} w(t)=\frac{1}{\nu}[\cos (\nu t)-1]
$$

Hence, we compute

$$
\|f\|_{\left[H_{, 0}^{1}(0, T)\right]^{\prime}}^{2}=\left\|\partial_{t} w\right\|_{L^{2}(0, T)}^{2}=\frac{1}{2} \frac{1}{\nu^{3}}[3 \nu T+\cos (\nu T) \sin (\nu T)-4 \sin (\nu T)] \simeq \frac{3}{2} \frac{T}{\nu^{2}}
$$

as $\nu \rightarrow \infty$. In particular, we have

$$
\frac{\left\|\partial_{t} u\right\|_{L^{2}(0, T)}}{\|f\|_{\left[H_{, 0}^{1}(0, T)\right]^{\prime}}} \simeq \frac{1}{6} \nu T
$$

as $\nu \rightarrow \infty$, which shows that the estimate (4.9) is sharp with respect to the order of $\nu$ and $T$, respectively.

While for $f \in\left[H_{, 0}^{1}(0, T)\right]^{\prime}$, the bound (4.9) exhibits an explicit dependence on $\nu=\sqrt{\mu}$, we can prove an estimate independent of $\mu$ when assuming $f \in L^{2}(0, T)$.

LEMMA 4.5. For given $f \in L^{2}(0, T)$, the unique solution $u \in H_{0}^{1},(0, T)$ satisfies

$$
\|u\|_{H_{0}^{1}(0, T)}^{2}+\mu\|u\|_{L^{2}(0, T)}^{2} \leq \frac{1}{2} T^{2}\|f\|_{L^{2}(0, T)}^{2} .
$$

Proof. For the solution $u$ and its first-order derivative, we find the representations

$$
u(t)=\frac{1}{\nu} \int_{0}^{t} \sin (\nu(t-s)) f(s) d s
$$

and

$$
\partial_{t} u(t)=\int_{0}^{t} \cos (\nu(t-s)) f(s) d s
$$

Hence, we compute

$$
\begin{gathered}
{\left[\partial_{t} u(t)\right]^{2}+\nu^{2}[u(t)]^{2}=\left[\int_{0}^{t} \cos (\nu(t-s)) f(s) d s\right]^{2}+\left[\int_{0}^{t} \sin (\nu(t-s)) f(s) d s\right]^{2}} \\
\leq \int_{0}^{t} \cos ^{2}(\nu(t-s)) d s \int_{0}^{t}[f(s)]^{2} d s+\int_{0}^{t} \sin ^{2}(\nu(t-s)) d s \int_{0}^{t}[f(s)]^{2} d s \\
=t \int_{0}^{t}[f(s)]^{2} d s \leq t \int_{0}^{T}[f(s)]^{2} d s,
\end{gathered}
$$

and therefore we obtain

$$
\begin{aligned}
\|u\|_{H_{0}^{1}(0, T)}^{2}+\mu\|u\|_{L^{2}(0, T)}^{2} & =\int_{0}^{T}\left\{\left[\partial_{t} u(t)\right]^{2}+\mu[u(t)]^{2}\right\} d t \\
& \leq \int_{0}^{T} t d t \int_{0}^{T}[f(s)]^{2} d s=\frac{1}{2} T^{2}\|f\|_{L^{2}(0, T)}^{2} .
\end{aligned}
$$

REMARK 4.6. As in Remark 4.4, we consider problem (4.6) for $f(t)=\sin (\nu t)$ with the solution $u(t)$ and its derivative $\partial_{t} u(t)=\frac{1}{2} t \sin (\nu t)$, i.e.,

$$
\left\|\partial_{t} u\right\|_{L^{2}(0, T)}^{2} \simeq \frac{1}{24} T^{3}, \quad\|f\|_{L^{2}(0, T)}^{2}=\frac{1}{2} \frac{1}{\nu}[\nu T-\cos (\nu T) \sin (\nu T)] \simeq \frac{1}{2} T .
$$


Hence, we conclude

$$
\frac{\left\|\partial_{t} u\right\|_{L^{2}(0, T)}^{2}}{\|f\|_{L^{2}(0, T)}^{2}} \simeq \frac{1}{12} T^{2}
$$

i.e., the estimate (4.10) is sharp with respect to the order of $T$.

The Galerkin-Bubnov finite element formulation of the equivalent variational formulation (4.7) is to find $u_{h} \in V_{h}:=S_{h}^{1}(0, T) \cap H_{0}^{1}(0, T)$ such that

(4.11) $a\left(u_{h}, \overline{\mathcal{H}}_{T} v_{h}\right)=-\left\langle\partial_{t} u_{h}, \partial_{t} \overline{\mathcal{H}}_{T} v_{h}\right\rangle_{L^{2}(0, T)}+\mu\left\langle u_{h}, \overline{\mathcal{H}}_{T} v_{h}\right\rangle_{L^{2}(0, T)}=\left\langle f, \overline{\mathcal{H}}_{T} v_{h}\right\rangle_{(0, T)}$

is satisfied for all $v_{h} \in V_{h}$. Unique solvability and related error estimates follow as for the numerical solution of elliptic operator equations with compact perturbations, which is based on a discrete stability condition.

THEOREM 4.7. Let

$$
h \leq \frac{2 \sqrt{3}}{(2+\sqrt{\mu} T) \mu T}
$$

be satisfied. Then, the bilinear form $a(\cdot, \cdot)$ as defined in (4.7) satisfies the stability condition (4.13)

$$
\frac{4}{(2+\sqrt{\mu} T)^{2}(2+\mu T)}\left\|\partial_{t} u_{h}\right\|_{L^{2}(0, T)} \leq \sup _{\substack{0 \neq v_{h} \in V_{h}\\}} \frac{a\left(u, \overline{\mathcal{H}}_{T} v_{h}\right)}{\left\|\partial_{t} v_{h}\right\|_{L^{2}(0, T)}} \quad \text { for all } u_{h} \in V_{h} .
$$

Proof. For $u_{h} \in V_{h}$, we define $w \in H_{0,}^{1}(0, T)$ as the unique solution of the variational problem

$$
-\int_{0}^{T} \partial_{t} w(t) \partial_{t}\left(\overline{\mathcal{H}}_{T} v\right)(t) d t=-\mu \int_{0}^{T} u_{h}(t)\left(\overline{\mathcal{H}}_{T} v\right)(t) d t \quad \text { for all } v \in H_{0,}^{1}(0, T)
$$

i.e., $w \in H_{0}^{1},(0, T)$ is the weak solution of the initial value problem

$$
\partial_{t t} w(t)=-\mu u_{h}(t) \quad \text { for } t \in(0, T), \quad w(0)=\partial_{t} w(0)=0 .
$$

Then, by using $\left(\overline{\mathcal{H}}_{T} v\right)(t)=v(T)-v(t)$,

$$
\begin{aligned}
a\left(u_{h}, \overline{\mathcal{H}}_{T}\left(u_{h}-w\right)\right)=-\int_{0}^{T} \partial_{t} u_{h}(t) \partial_{t}\left[\left(\overline{\mathcal{H}}_{T} u_{h}\right)(t)-\left(\overline{\mathcal{H}}_{T} w\right)(t)\right] d t \\
\quad+\mu \int_{0}^{T} u_{h}(t)\left[\left(\overline{\mathcal{H}}_{T} u_{h}\right)(t)-\left(\overline{\mathcal{H}}_{T} w\right)(t)\right] d t \\
=\int_{0}^{T} \partial_{t} u_{h}(t)\left[\partial_{t} u_{h}(t)-\partial_{t} w(t)\right] d t-\int_{0}^{T} \partial_{t} w(t)\left[\partial_{t} u_{h}(t)-\partial_{t} w(t)\right] d t \\
=\int_{0}^{T}\left[\partial_{t} u_{h}(t)-\partial_{t} w(t)\right]^{2} d t .
\end{aligned}
$$

In addition, let $z \in H_{0}^{1}(0, T)$ be the unique solution of the variational formulation such that (4.15)

$$
-\int_{0}^{T} \partial_{t} z(t) \partial_{t}\left(\overline{\mathcal{H}}_{T} v\right)(t) d t=-\int_{0}^{T} \partial_{t} u_{h}(t) \partial_{t}\left(\overline{\mathcal{H}}_{T} v\right)(t) d t+\mu \int_{0}^{T} u_{h}(t)\left(\overline{\mathcal{H}}_{T} v\right)(t) d t
$$


is satisfied for all $v \in H_{0}^{1}(0, T)$. With (4.14) this is equivalent to

$$
-\int_{0}^{T} \partial_{t}\left[z(t)-\left(u_{h}(t)-w(t)\right)\right] \partial_{t}\left(\overline{\mathcal{H}}_{T} v\right)(t) d t=0 \quad \text { for all } v \in H_{0,}^{1}(0, T),
$$

from which we conclude, recalling $u_{h}(0)=w(0)=z(0)=0$, that

$$
z(t)=u_{h}(t)-w(t)
$$

i.e., we have

$$
a\left(u_{h}, \overline{\mathcal{H}}_{T}\left(u_{h}-w\right)\right)=\left\|\partial_{t} z\right\|_{L^{2}(0, T)}^{2} .
$$

On the other hand, the variational formulation (4.15) gives

$$
\begin{aligned}
\left\|\partial_{t} z\right\|_{L^{2}(0, T)}=\frac{a\left(u_{h}, \overline{\mathcal{H}}_{T} z\right)}{\left\|\partial_{t} z\right\|_{L^{2}(0, T)}} & \leq \sup _{0 \neq v \in H_{0,(0, T)}^{1}} \frac{a\left(u_{h}, \overline{\mathcal{H}}_{T} v\right)}{\left\|\partial_{t} v\right\|_{L^{2}(0, T)}} \\
& =\sup _{0 \neq v \in H_{0,(0, T)}^{1}} \frac{\left\langle\partial_{t} z, \partial_{t} v\right\rangle_{L^{2}(0, T)}}{\left\|\partial_{t} v\right\|_{L^{2}(0, T)}} \leq\left\|\partial_{t} z\right\|_{L^{2}(0, T)},
\end{aligned}
$$

i.e.,

$$
\left\|\partial_{t} z\right\|_{L^{2}(0, T)}=\sup _{0 \neq v \in H_{0,(0, T)}^{1}} \frac{a\left(u_{h}, \overline{\mathcal{H}}_{T} v\right)}{\left\|\partial_{t} v\right\|_{L^{2}(0, T)}} \geq \frac{2}{2+\nu T}\left\|\partial_{t} u_{h}\right\|_{L^{2}(0, T)}
$$

when using (4.8). With this, we conclude

$$
a\left(u_{h}, \overline{\mathcal{H}}_{T}\left(u_{h}-w\right)\right) \geq \frac{4}{(2+\nu T)^{2}}\left\|\partial_{t} u_{h}\right\|_{L^{2}(0, T)}^{2} .
$$

According to (4.14), we define $w_{h} \in V_{h}$ as the unique solution of

$$
\int_{0}^{T} \partial_{t} w_{h}(t) \partial_{t} v_{h}(t) d t=-\mu \int_{0}^{T} u_{h}(t)\left(\overline{\mathcal{H}}_{T} v_{h}\right)(t) d t \quad \text { for all } v_{h} \in V_{h} .
$$

Then, there holds true the Galerkin orthogonality

$$
\int_{0}^{T}\left[\partial_{t} w(t)-\partial_{t} w_{h}(t)\right] \partial_{t} v_{h}(t) d t=0 \quad \text { for all } v_{h} \in V_{h},
$$

and by using Céa's lemma and standard interpolation error estimates, the error estimate

$$
\begin{aligned}
& \left\|\partial_{t} w-\partial_{t} w_{h}\right\|_{L^{2}(0, T)} \leq \inf _{v_{h} \in V_{h}}\left\|\partial_{t} w-\partial_{t} v_{h}\right\|_{L^{2}(0, T)} \\
& \leq\left\|\partial_{t}\left(w-I_{h} w\right)\right\|_{L^{2}(0, T)} \leq \frac{1}{\sqrt{3}} h\left\|\partial_{t t} w\right\|_{L^{2}(0, T)}=\frac{1}{\sqrt{3}} \mu h\left\|u_{h}\right\|_{L^{2}(0, T)}
\end{aligned}
$$

follows. With this, we have

$$
\begin{aligned}
a\left(u_{h}, \overline{\mathcal{H}}_{T}\left(w-w_{h}\right)\right) & =\int_{0}^{T} \partial_{t} u_{h}(t) \partial_{t}\left(w(t)-w_{h}(t)\right) d t+\mu \int_{0}^{T} u_{h}(t)\left(\overline{\mathcal{H}}_{T}\left(w-w_{h}\right)\right)(t) d t \\
& =\mu \int_{0}^{T} u_{h}(t)\left(\overline{\mathcal{H}}_{T}\left(w-w_{h}\right)\right)(t) d t \\
& \leq \mu\left\|u_{h}\right\|_{L^{2}(0, T)}\left\|\overline{\mathcal{H}}_{T}\left(w-w_{h}\right)\right\|_{L^{2}(0, T)} .
\end{aligned}
$$


Next, we define $\psi \in H_{0}^{1}(0, T)$ as the unique solution of the variational formulation

$$
-\int_{0}^{T} \partial_{t} \psi(t) \partial_{t}\left(\overline{\mathcal{H}}_{T} v\right)(t) d t=\int_{0}^{T}\left(\overline{\mathcal{H}}_{T}\left(w-w_{h}\right)\right)(t)\left(\overline{\mathcal{H}}_{T} v\right)(t) d t \quad \text { for all } v \in H_{0}^{1}(0, T),
$$

i.e.,

$$
\partial_{t t} \psi(t)=\left(\overline{\mathcal{H}}_{T}\left(w-w_{h}\right)\right)(t) \quad \text { for } t \in(0, T), \quad \psi(0)=\partial_{t} \psi(t)=0 .
$$

In particular for $v=w-w_{h} \in H_{0}^{1}(0, T)$, we conclude

$$
\begin{aligned}
\left\|\overline{\mathcal{H}}_{T}\left(w-w_{h}\right)\right\|_{L^{2}(0, T)}^{2} & =\int_{0}^{T}\left(\overline{\mathcal{H}}_{T}\left(w-w_{h}\right)\right)(t)\left(\overline{\mathcal{H}}_{T}\left(w-w_{h}\right)\right)(t) d t \\
& =-\int_{0}^{T} \partial_{t} \psi(t) \partial_{t}\left(\overline{\mathcal{H}}_{T}\left(w-w_{h}\right)\right)(t) d t \\
& =\int_{0}^{T} \partial_{t} \psi(t)\left[\partial_{t} w(t)-\partial_{t} w_{h}(t)\right] d t \\
& =\int_{0}^{T} \partial_{t}\left[\psi(t)-I_{h} \psi(t)\right]\left[\partial_{t} w(t)-\partial_{t} w_{h}(t)\right] d t \\
& \leq\left\|\partial_{t}\left(\psi-I_{h} \psi\right)\right\|_{L^{2}(0, T)}\left\|\partial_{t}\left(w-w_{h}\right)\right\|_{L^{2}(0, T)} \\
& \leq \frac{1}{3} h^{2}\left\|\partial_{t t} \psi\right\|_{L^{2}(0, T)}\left\|\partial_{t t} w\right\|_{L^{2}(0, T)} \\
& =\frac{1}{3} \mu h^{2}\left\|\overline{\mathcal{H}}_{T}\left(w-w_{h}\right)\right\|_{L^{2}(0, T)}\left\|u_{h}\right\|_{L^{2}(0, T)},
\end{aligned}
$$

i.e.,

$$
\left\|\overline{\mathcal{H}}_{T}\left(w-w_{h}\right)\right\|_{L^{2}(0, T)} \leq \frac{1}{3} \mu h^{2}\left\|u_{h}\right\|_{L^{2}(0, T)},
$$

and therefore, by using $u_{h} \in H_{0}^{1}(0, T)$,

$$
a\left(u_{h}, \overline{\mathcal{H}}_{T}\left(w-w_{h}\right)\right) \leq \frac{1}{3} \mu^{2} h^{2}\left\|u_{h}\right\|_{L^{2}(0, T)}^{2} \leq \frac{1}{6} \mu^{2} h^{2} T^{2}\left\|\partial_{t} u_{h}\right\|_{L^{2}(0, T)}^{2}
$$

follows. Hence, we conclude

$$
\begin{aligned}
a\left(u_{h}, \overline{\mathcal{H}}_{T}\left(u_{h}-w_{h}\right)\right) & =a\left(u_{h}, \overline{\mathcal{H}}_{T}\left(u_{h}-w\right)\right)+a\left(u_{h}, \overline{\mathcal{H}}_{T}\left(w-w_{h}\right)\right) \\
& \geq\left[\frac{4}{(2+\sqrt{\mu} T)^{2}}-\frac{1}{6} \mu^{2} h^{2} T^{2}\right]\left\|\partial_{t} u_{h}\right\|_{L^{2}(0, T)}^{2} \\
& \geq \frac{2}{(2+\sqrt{\mu} T)^{2}}\left\|\partial_{t} u_{h}\right\|_{L^{2}(0, T)}^{2}
\end{aligned}
$$

if

$$
\frac{1}{6} \mu^{2} h^{2} T^{2} \leq \frac{2}{(2+\sqrt{\mu} T)^{2}}
$$

is satisfied, i.e.,

$$
h^{2} \leq \frac{12}{(2+\sqrt{\mu} T)^{2} \mu^{2} T^{2}} .
$$


Finally, we have

$$
\left\|\partial_{t}\left(u_{h}-w_{h}\right)\right\|_{L^{2}(0, T)} \leq\left\|\partial_{t} u_{h}\right\|_{L^{2}(0, T)}+\left\|\partial_{t} w_{h}\right\|_{L^{2}(0, T)}
$$

and

$$
\begin{aligned}
\left\|\partial_{t} w_{h}\right\|_{L^{2}(0, T)}^{2} & =-\int_{0}^{T} \partial_{t} w_{h}(t) \partial_{t}\left(\overline{\mathcal{H}}_{T} w_{h}\right)(t) d t=-\mu \int_{0}^{T} u_{h}(t)\left(\overline{\mathcal{H}}_{T} w_{h}\right)(t) d t \\
& \leq \mu\left\|u_{h}\right\|_{L^{2}(0, T)}\left\|\overline{\mathcal{H}}_{T} w_{h}\right\|_{L^{2}(0, T)} \leq \frac{1}{2} \mu T\left\|\partial_{t} u_{h}\right\|_{L^{2}(0, T)}\left\|\partial_{t} w_{h}\right\|_{L^{2}(0, T)},
\end{aligned}
$$

i.e.,

$$
\left\|\partial_{t}\left(u_{h}-w_{h}\right)\right\|_{L^{2}(0, T)} \leq\left(1+\frac{1}{2} \mu T\right)\left\|\partial_{t} u_{h}\right\|_{L^{2}(0, T)} .
$$

This concludes the proof.

For any $w \in H_{0,}^{1}(0, T)$, we define $w_{h}=G_{h} w \in V_{h}$ as the Galerkin projection satisfying

$$
a\left(G_{h} w, \overline{\mathcal{H}}_{T} v_{h}\right)=a\left(w, \overline{\mathcal{H}}_{T} v_{h}\right) \quad \text { for all } v_{h} \in V_{h},
$$

where the stability condition (4.13) implies

$$
\begin{aligned}
& \frac{4}{(2+\sqrt{\mu} T)^{2}(2+\mu T)}\left\|\partial_{t} G_{h} w\right\|_{L^{2}(0, T)} \leq \sup _{0 \neq v_{h} \in V_{h}} \frac{a\left(G_{h} w, \overline{\mathcal{H}}_{T} v_{h}\right)}{\left\|\partial_{t} v_{h}\right\|_{L^{2}(0, T)}} \\
& =\sup _{0 \neq v_{h} \in V_{h}} \frac{a\left(w, \overline{\mathcal{H}}_{T} v_{h}\right)}{\left\|\partial_{t} v_{h}\right\|_{L^{2}(0, T)}} \\
& \leq \sup _{\substack{0 \neq v_{h} \in V_{h}\\
}} \frac{\left\|\partial_{t} w\right\|_{L^{2}(0, T)}\left\|\partial_{t} v_{h}\right\|_{L^{2}(0, T)}+\mu\|w\|_{L^{2}(0, T)}\left\|\overline{\mathcal{H}}_{T} v_{h}\right\|_{L^{2}(0, T)}}{\left\|\partial_{t} v_{h}\right\|_{L^{2}(0, T)}} \\
& \leq\left(1+\frac{1}{2} \mu T\right)\left\|\partial_{t} w\right\|_{L^{2}(0, T)}
\end{aligned}
$$

i.e.,

$$
\left\|\partial_{t} G_{h} w\right\|_{L^{2}(0, T)} \leq \frac{1}{8}(2+\sqrt{\mu} T)^{2}(2+\mu T)^{2}\left\|\partial_{t} w\right\|_{L^{2}(0, T)} \quad \text { for all } w \in H_{0,}^{1}(0, T) .
$$

So, we are in a position to state a convergence result for the finite element solution $u_{h}$ of the variational formulation (4.7).

THEOREM 4.8. Let $u \in H_{0}^{1}(0, T)$ and $u_{h} \in V_{h} \subset H_{0,}^{1}(0, T)$ be the unique solutions of the variational formulations (4.7) and (4.11), respectively. We assume $u \in H^{2}(0, T)$, and let (4.12) be satisfied. Then, there holds true the error estimate

$$
\left\|\partial_{t}\left(u-u_{h}\right)\right\|_{L^{2}(0, T)} \leq \frac{1}{\sqrt{3}}\left[1+\frac{1}{8}(2+\sqrt{\mu} T)^{2}(2+\mu T)^{2}\right] h\left\|\partial_{t t} u\right\|_{L^{2}(0, T)} .
$$

Proof. With $u_{h}=G_{h} u$ and $v_{h}=G_{h} v_{h}$ for all $v_{h} \in V_{h}$, we have by Céa's lemma,

$$
\begin{aligned}
\left\|\partial_{t}\left(u-u_{h}\right)\right\|_{L^{2}(0, T)} & \leq\left\|\partial_{t}\left(u-v_{h}\right)\right\|_{L^{2}(0, T)}+\left\|\partial_{t} G_{h}\left(u-v_{h}\right)\right\|_{L^{2}(0, T)} \\
& \leq\left[1+\frac{1}{8}(2+\sqrt{\mu} T)^{2}(2+\mu T)^{2}\right]\left\|\partial_{t}\left(u-v_{h}\right)\right\|_{L^{2}(0, T)}
\end{aligned}
$$

for all $v_{h} \in V_{h}$, and the assertion follows from standard interpolation error estimates. 
For the discretisation of the variational formulation (4.11), we use the same notation as in Section 2 to find $u_{h} \in V_{h}=\operatorname{span}\left\{\varphi_{k}\right\}_{k=1}^{N}$ such that

$$
-\int_{0}^{T} \partial_{t} u_{h}(t) \partial_{t} \varphi_{\ell}(t) d t+\mu \int_{0}^{T} u_{h}(t) \varphi_{\ell}(t) d t=\int_{0}^{T} f(t) \varphi_{\ell}(t) d t=: f_{\ell}
$$

is satisfied for all $\ell=0, \ldots, N-1$. Since we consider a uniform discretisation, the stiffness matrix is given by

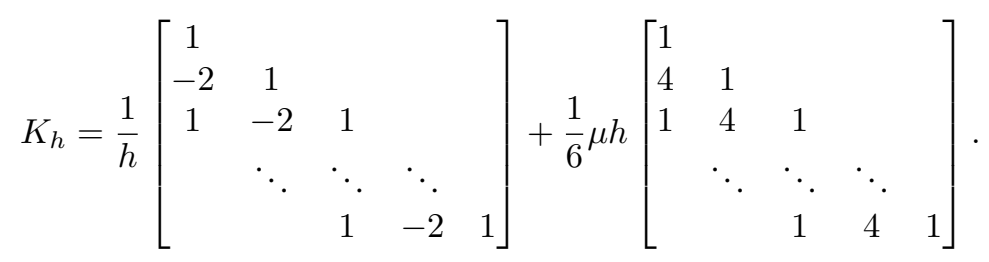

Obviously, we have unique solvability of (4.17) for all $\mu$ independent of $h$.

REMARK 4.9. For $\ell=2, \ldots, N-1$, we can write the finite element formulation (4.17) as

$$
\left(\frac{1}{h}+\frac{1}{6} \mu h\right) u_{\ell-1}+\left(\frac{2}{3} \mu h-\frac{2}{h}\right) u_{\ell}+\left(\frac{1}{h}+\frac{1}{6} \mu h\right) u_{\ell+1}=f_{\ell}
$$

which is a kind of a two-step method [18, Chapter III.2]. This method is zero-stable if and only if the root condition [18, Chapter III.3] is satisfied. For (4.18), we therefore obtain the condition

$$
\mu h^{2}<12 .
$$

For the numerical example, we consider again the solution $u(t)=\sin ^{2}\left(\frac{5}{4} \pi t\right)$ for $t \in(0, T)$ with $T=2$ and $\mu=10$. The numerical results are given in Table 4.2, where we observe linear convergence in the energy norm as predicted in (4.16) and second-order convergence in $L^{2}(0, T)$, which can be proven when applying the Nitsche trick. In Table 4.3 we present the related numerical results for the case $\mu=1000$. We observe convergence only when $h$ is sufficiently small. According to (4.19), we note that $\sqrt{12 / \mu} \approx 0.1095$. So, it remains open to improve assumption (4.12) to ensure the stability condition (4.13). On the other hand, following [47], it is possible to derive and to analyse a stabilised variational formulation for the initial value problem (4.6); see [38]. Using the $L^{2}$-projection $Q_{h}^{0}$ on the finite element space $S_{h}^{0}(0, T)$ of piecewise constant functions, we may consider, instead of (4.11), the perturbed variational problem to find $\widetilde{u}_{h} \in S_{h}^{1}(0, T) \cap H_{0,}^{1}(0, T)$ such that

$$
-\left\langle\partial_{t} \widetilde{u}_{h}, \partial_{t} w_{h}\right\rangle_{L^{2}(0, T)}+\mu\left\langle\widetilde{u}_{h}, Q_{h}^{0} w_{h}\right\rangle_{L^{2}(0, T)}=\left\langle f, w_{h}\right\rangle_{(0, T)}
$$

is satisfied for all $w_{h} \in S_{h}^{1}(0, T) \cap H_{, 0}^{1}(0, T)$. The stability and error analysis of (4.20) is based on a discrete inf-sup condition [38, Lemma 17.6], which then results in an optimal energy error estimate [38, Theorem 17.1].

5. The wave equation. As model problem for a hyperbolic partial differential equation, we consider the Dirichlet problem for the wave equation,

$$
\begin{aligned}
\partial_{t t} u(x, t)-\Delta_{x} u(x, t) & =f(x, t) & & \text { for }(x, t) \in Q:=\Omega \times(0, T), \\
u(x, t) & =0 & & \text { for }(x, t) \in \Sigma:=\Gamma \times(0, T), \\
u(x, 0)=\partial_{t} u(x, 0) & =0 & & \text { for } x \in \Omega,
\end{aligned}
$$




\section{ETNA}

Kent State University and

Johann Radon Institute (RICAM)

188

O. STEINBACH AND M. ZANK

TABLE 4.2

Numerical results for the Galerkin-Petrov formulation (4.17), $\mu=10$.

\begin{tabular}{rccccr}
$N$ & $h$ & $\left\|u-u_{h}\right\|_{L^{2}}$ & eoc & $\left\|\partial_{t}\left(u-u_{h}\right)\right\|_{L^{2}}$ & eoc \\
\hline 4 & 0.5000000 & $5.3407 \mathrm{e}-01$ & - & $3.5365 \mathrm{e}+00$ & - \\
8 & 0.2500000 & $1.7632 \mathrm{e}-01$ & 1.6 & $2.1021 \mathrm{e}+00$ & 0.8 \\
16 & 0.1250000 & $4.9649 \mathrm{e}-02$ & 1.8 & $1.0979 \mathrm{e}+00$ & 0.9 \\
32 & 0.0625000 & $1.2804 \mathrm{e}-02$ & 2.0 & $5.5462 \mathrm{e}-01$ & 1.0 \\
64 & 0.0312500 & $3.2263 \mathrm{e}-03$ & 2.0 & $2.7800 \mathrm{e}-01$ & 1.0 \\
128 & 0.0156250 & $8.0816 \mathrm{e}-04$ & 2.0 & $1.3909 \mathrm{e}-01$ & 1.0 \\
256 & 0.0078125 & $2.0214 \mathrm{e}-04$ & 2.0 & $6.9555 \mathrm{e}-02$ & 1.0 \\
512 & 0.0039062 & $5.0541 \mathrm{e}-05$ & 2.0 & $3.4779 \mathrm{e}-02$ & 1.0 \\
1024 & 0.0019531 & $1.2636 \mathrm{e}-05$ & 2.0 & $1.7390 \mathrm{e}-02$ & 1.0 \\
2048 & 0.0009766 & $3.1589 \mathrm{e}-06$ & 2.0 & $8.6948 \mathrm{e}-03$ & 1.0 \\
4096 & 0.0004883 & $7.8972 \mathrm{e}-07$ & 2.0 & $4.3474 \mathrm{e}-03$ & 1.0 \\
8192 & 0.0002441 & $1.9737 \mathrm{e}-07$ & 2.0 & $2.1737 \mathrm{e}-03$ & 1.0
\end{tabular}

TABLE 4.3

Numerical results for the Galerkin-Petrov formulation (4.17), $\mu=1000$.

\begin{tabular}{rccrcr}
$N$ & $h$ & $\left\|u-u_{h}\right\|_{L^{2}}$ & eoc & $\left\|\partial_{t}\left(u-u_{h}\right)\right\|_{L^{2}}$ & eoc \\
\hline 4 & 0.5000000 & $8.0288 \mathrm{e}+00$ & - & $4.1323 \mathrm{e}+01$ & - \\
8 & 0.2500000 & $2.3961 \mathrm{e}+02$ & -4.9 & $2.4811 \mathrm{e}+03$ & -5.9 \\
16 & 0.1250000 & $4.4282 \mathrm{e}+01$ & 2.4 & $1.1065 \mathrm{e}+03$ & 1.2 \\
32 & 0.0625000 & $9.5909 \mathrm{e}-03$ & 12.2 & $6.2095 \mathrm{e}-01$ & 10.8 \\
64 & 0.0312500 & $2.7371 \mathrm{e}-03$ & 1.8 & $2.8953 \mathrm{e}-01$ & 1.1 \\
128 & 0.0156250 & $7.1356 \mathrm{e}-04$ & 1.9 & $1.4072 \mathrm{e}-01$ & 1.0 \\
256 & 0.0078125 & $1.8124 \mathrm{e}-04$ & 2.0 & $6.9765 \mathrm{e}-02$ & 1.0 \\
512 & 0.0039062 & $4.5486 \mathrm{e}-05$ & 2.0 & $3.4805 \mathrm{e}-02$ & 1.0 \\
1024 & 0.0019531 & $1.1382 \mathrm{e}-05$ & 2.0 & $1.7393 \mathrm{e}-02$ & 1.0 \\
2048 & 0.0009766 & $2.8463 \mathrm{e}-06$ & 2.0 & $8.6952 \mathrm{e}-03$ & 1.0 \\
4096 & 0.0004883 & $7.1162 \mathrm{e}-07$ & 2.0 & $4.3474 \mathrm{e}-03$ & 1.0 \\
8192 & 0.0002441 & $1.7791 \mathrm{e}-07$ & 2.0 & $2.1737 \mathrm{e}-03$ & 1.0
\end{tabular}

where $\Omega \subset \mathbb{R}^{d}, d=1,2,3$, is a bounded domain with, for $d=2,3$, Lipschitz boundary $\Gamma=\partial \Omega$. According to the previous sections, we consider the variational formulation of (5.1) to find $u \in H_{0 ; 0}^{1,1}(Q):=L^{2}\left(0, T ; H_{0}^{1}(\Omega)\right) \cap H_{0,}^{1}\left(0, T ; L^{2}(\Omega)\right)$ such that

$$
-\left\langle\partial_{t} u, \partial_{t} v\right\rangle_{L^{2}(Q)}+\left\langle\nabla_{x} u, \nabla_{x} v\right\rangle_{L^{2}(Q)}=\langle f, v\rangle_{L^{2}(Q)}
$$

is satisfied for all $v \in H_{0 ;, 0}^{1,1}(Q):=L^{2}\left(0, T ; H_{0}^{1}(\Omega)\right) \cap H_{, 0}^{1}\left(0, T ; L^{2}(\Omega)\right)$. Note that the initial condition $u(\cdot, 0)=0$ is considered in the strong sense, whereas the initial condition $\partial_{t} u(\cdot, 0)=0$ is incorporated in a weak sense. For $u \in H_{0 ; 0}^{1,1}(Q)$, an appropriate norm is given by

$$
\|u\|_{H_{0 ; 0}^{1,1}(Q)}^{2}=\int_{0}^{T} \int_{\Omega}\left[\left|\partial_{t} u(x, t)\right|^{2}+\left|\nabla_{x} u(x, t)\right|^{2}\right] d x d t .
$$


As in [22], we state the following result on the unique solvability of the variational formulation (5.2) when assuming $f \in L^{2}(Q)$.

THEOREM 5.1. For $f \in L^{2}(Q)$, there exists a unique solution $u \in H_{0 ; 0}^{1,1}(Q)$ of the variational formulation (5.2) satisfying

$$
\|u\|_{H_{0 ; 0}^{1,1}(Q)} \leq \frac{1}{\sqrt{2}} T\|f\|_{L^{2}(Q)}
$$

Proof. When using the representation (3.2), any $u \in H_{0 ; 0}^{1,1}(Q)$ can be written as

$$
u(x, t)=\sum_{i=1}^{\infty} \sum_{k=0}^{\infty} u_{i, k} v_{k}(t) \phi_{i}(x)=\sum_{i=1}^{\infty} U_{i}(t) \phi_{i}(x)
$$

where $v_{k}(t)$ are the temporal eigenfunctions as given in (2.5), and $\phi_{i}(x)$ are the spatial $L^{2}(\Omega)$ orthonormal eigenfunctions of the Laplacian with homogeneous Dirichlet boundary conditions. For the solution of the variational problem (5.2), we use the ansatz (5.3), where the functions $U_{i} \in H_{0,}^{1}(0, T)$ are unknown functions to be determined. When choosing, for a fixed $j \in \mathbb{N}$, $v(x, t)=V(t) \phi_{j}(x)$ with $V \in H_{, 0}^{1}(0, T)$ as test function, the variational formulation (5.2) results in finding $U_{j} \in H_{0}^{1},(0, T)$ such that

$$
-\int_{0}^{T} \partial_{t} U_{j}(t) \partial_{t} V(t) d t+\mu_{j} \int_{0}^{T} U_{j}(t) V(t) d t=\int_{0}^{T} f_{j}(t) V(t) d t
$$

is satisfied for all $V \in H_{, 0}^{1}(0, T)$, where

$$
f_{j}(t)=\int_{\Omega} f(x, t) \phi_{j}(x) d x
$$

are the coefficients of the Fourier expansion

$$
f(x, t)=\sum_{j=1}^{\infty} f_{j}(t) \phi_{j}(x) .
$$

From this, we conclude

$$
\begin{aligned}
\|f\|_{L^{2}(Q)}^{2} & =\int_{0}^{T} \int_{\Omega}[f(x, t)]^{2} d x d t=\sum_{i=1}^{\infty} \sum_{j=1}^{\infty} \int_{0}^{T} f_{i}(t) f_{j}(t) d t \int_{\Omega} \phi_{i}(x) \phi_{j}(x) d x \\
& =\sum_{j=1}^{\infty} \int_{0}^{T}\left[f_{j}(t)\right]^{2} d t=\sum_{j=1}^{\infty}\left\|f_{j}\right\|_{L^{2}(0, T)}^{2},
\end{aligned}
$$


and hence we obtain by using (4.10),

$$
\begin{aligned}
\|u\|_{H_{0 ; 0}^{1,1}(Q)}^{2}= & \int_{0}^{T} \int_{\Omega}\left[\left|\partial_{t} u(x, t)\right|^{2}+\left|\nabla_{x} u(x, t)\right|^{2}\right] d x d t \\
= & \sum_{i=1}^{\infty} \sum_{j=1}^{\infty}\left[\int_{0}^{T} \partial_{t} U_{i}(t) \partial_{t} U_{j}(t) d t \int_{\Omega} \phi_{i}(x) \phi_{j}(x) d x\right. \\
& \left.\quad+\int_{0}^{T} U_{i}(t) U_{j}(t) d t \int_{\Omega} \nabla_{x} \phi_{i}(x) \cdot \nabla_{x} \phi_{j}(x) d x\right] \\
= & \sum_{i=1}^{\infty}\left[\int_{0}^{T}\left|\partial_{t} U_{i}(t)\right|^{2} d t+\mu_{i} \int_{0}^{T}\left|U_{i}(t)\right|^{2} d t\right] \\
= & \sum_{i=1}^{\infty}\left[\left\|U_{i}\right\|_{H_{0,}^{1}(0, T)}^{2}+\mu_{i}\left\|U_{i}\right\|_{L^{2}(0, T)}^{2}\right] \\
\leq & \frac{1}{2} T^{2} \sum_{i=1}^{\infty}\left\|f_{i}\right\|_{L^{2}(0, T)}^{2}=\frac{1}{2} T^{2}\|f\|_{L^{2}(Q)}^{2} .
\end{aligned}
$$

The variational formulation (5.2) is equivalent to finding $u \in H_{0 ; 0}^{1,1}(Q)$ such that

$$
-\left\langle\partial_{t} u, \partial_{t} \overline{\mathcal{H}}_{T} v\right\rangle_{L^{2}(Q)}+\left\langle\nabla_{x} u, \nabla_{x} \overline{\mathcal{H}}_{T} v\right\rangle_{L^{2}(Q)}=\left\langle f, \overline{\mathcal{H}}_{T} v\right\rangle_{L^{2}(Q)}
$$

is satisfied for all $v \in H_{0 ; 0}^{1,1}(Q)$, where the transformation operator $\overline{\mathcal{H}}_{T}$ acts only on the time variable $t$.

As in the case of the heat equation, we consider the tensor-product space-time finite element space $\mathcal{V}_{h}=W_{h_{x}} \otimes V_{h_{t}} \subset H_{0 ; 0}^{1,1}(Q)$ with piecewise linear, continuous functions $W_{h_{x}}=\operatorname{span}\left\{\psi_{i}\right\}_{i=1}^{M_{x}} \subset H_{0}^{1}(\Omega)$ and $V_{h_{t}}=S_{h_{t}}^{1}(0, T) \cap H_{0}^{1}(0, T)=\operatorname{span}\left\{\varphi_{k}\right\}_{k=1}^{N_{t}}$. Then, the Galerkin-Bubnov finite element discretisation of the variational formulation (5.4) is to find $u_{h} \in \mathcal{V}_{h}$ such that

$$
-\left\langle\partial_{t} u_{h}, \partial_{t} \overline{\mathcal{H}}_{T} v_{h}\right\rangle_{L^{2}(Q)}+\left\langle\nabla_{x} u_{h}, \nabla_{x} \overline{\mathcal{H}}_{T} v_{h}\right\rangle_{L^{2}(Q)}=\left\langle f, \overline{\mathcal{H}}_{T} v_{h}\right\rangle_{L^{2}(Q)}
$$

is satisfied for all $v_{h} \in \mathcal{V}_{h}$. Recall that the transformation $\overline{\mathcal{H}}_{T} \varphi_{k}$ is realised by using (4.5). Since we are using a tensor-product space-time finite element space $\mathcal{V}_{h}=W_{h_{x}} \otimes V_{h_{t}}$, we can write

$$
u_{h}(x, t)=\sum_{k=1}^{N_{t}} \sum_{i=1}^{M_{x}} u_{i, k} \varphi_{k}(t) \psi_{i}(x)=\sum_{i=1}^{M_{x}} U_{i, h}(t) \psi_{i}(x), \quad U_{i, h}(t)=\sum_{k=1}^{N_{t}} u_{i, k} \varphi_{k}(t) .
$$

By using

$$
\widetilde{u}(x, t)=\sum_{i=1}^{M_{x}} \widetilde{U}_{i}(t) \psi_{i}(x),
$$

we can write the intermediate step of the semi-discretisation approach for solving (5.1) as

$$
M_{h} \partial_{t t} \underline{\widetilde{U}}(t)+K_{h} \underline{\tilde{U}}(t)=\underline{f}(t) \quad \text { for } t \in(0, T), \quad \underline{\widetilde{U}}(0)=\partial_{t} \underline{\widetilde{U}}(0)=\underline{0},
$$


with the spatial finite element mass matrix $M_{h}$, the stiffness matrix $K_{h}$, and the load vector $\underline{f}(t)$, i.e., for $i, j=1, \ldots, M_{x}$,

$$
\begin{aligned}
M_{h}[j, i] & =\int_{\Omega} \psi_{i}(x) \psi_{j}(x) d x, \\
K_{h}[j, i] & =\int_{\Omega} \nabla_{x} \psi_{i}(x) \cdot \nabla_{x} \psi_{j}(x) d x, \\
f_{j}(t) & =\int_{\Omega} f(x, t) \psi_{j}(x) d x .
\end{aligned}
$$

By using

$$
M_{h}=L_{h} L_{h}^{\top}, \quad A_{h}=L_{h}^{-1} K_{h} L_{h}^{-\top}, \quad \underline{W}=L_{h}^{\top} \underline{\widetilde{U}}, \quad \underline{g}(t)=L_{h}^{-1} \underline{f}(t),
$$

we further obtain

$$
\partial_{t t} \underline{W}(t)+A_{h} \underline{W}(t)=\underline{g}(t) \quad \text { for } t \in(0, T), \quad \underline{W}(0)=\partial_{t} \underline{W}(0)=\underline{0} .
$$

Since $A_{h}$ is symmetric and positive definite, we conclude the diagonal representation

$$
\begin{aligned}
A_{h} & =V_{h} D_{h} V_{h}^{\top}, & D_{h} & =\operatorname{diag}\left(\lambda_{i}\left(A_{h}\right)\right)_{i=1}^{M_{x}}, \\
V_{h} & =\left(\underline{v}^{1}, \ldots, \underline{v}^{M_{x}}\right), & A_{h} \underline{v}^{i} & =\lambda_{i}\left(A_{h}\right) \underline{v}^{i} .
\end{aligned}
$$

Finally, by using $\underline{Z}(t):=V_{h}^{\top} \underline{W}(t)$, we have to solve

$$
\partial_{t t} \underline{Z}(t)+D_{h} \underline{Z}(t)=V_{h}^{\top} \underline{g}(t)=: \underline{\widetilde{g}}(t) \quad \text { for } t \in(0, T), \quad \underline{Z}(0)=\partial_{t} \underline{Z}(0)=\underline{0},
$$

which consists of $M_{x}$ scalar equations of the form (4.6). The related finite element solution is defined by finding, for $i=1, \ldots, M_{x}, z_{i, h_{t}} \in V_{h_{t}}=S_{h_{t}}^{1}(0, T) \cap H_{0,}^{1}(0, T)$ such that

$$
-\left\langle\partial_{t} z_{i, h_{t}}, \partial_{t} \overline{\mathcal{H}}_{T} v_{h_{t}}\right\rangle_{L^{2}(0, T)}+\lambda_{i}\left(A_{h}\right)\left\langle z_{i, h_{t}}, \overline{\mathcal{H}}_{T} v_{h_{t}}\right\rangle_{L^{2}(0, T)}=\left\langle\widetilde{g}_{i}, \overline{\mathcal{H}}_{T} v_{h_{t}}\right\rangle_{(0, T)}
$$

is satisfied for all $v_{h_{t}} \in V_{h_{t}}$. By construction, we have

$$
\underline{Z}_{h}(t)=V_{h}^{\top} L_{h}^{\top} \underline{U}_{h}(t),
$$

where

$$
\underline{U}_{h}(t)=\left(U_{1, h}(t), \ldots, U_{M_{x}, h}(t)\right)^{\top}
$$

is the vector of the unknown functions of the approximation $u_{h}(x, t)$.

Stability and related error estimates for the finite element solutions $z_{i, h_{t}}$ follow for sufficiently small time mesh sizes $h_{t}$; see Theorem 4.8. However, as in Remark 4.9, we have stability, when the condition (4.19) is satisfied, i.e.,

$$
\lambda_{i}\left(A_{h}\right)=\frac{\left(A_{h} \underline{v}^{i}, \underline{v}^{i}\right)}{\left(\underline{v}^{i}, \underline{v}^{i}\right)}=\frac{\left(K_{h} \underline{u}^{i}, \underline{u}^{i}\right)}{\left(M_{h} \underline{u}^{i}, \underline{u}^{i}\right)}=\frac{\left\|\nabla_{x} u_{h}^{i}\right\|_{L^{2}(\Omega)}^{2}}{\left\|u_{h}^{i}\right\|_{L^{2}(\Omega)}^{2}}<\frac{12}{h_{t}^{2}} \quad \text { for } i=1, \ldots, M_{x},
$$

where $\underline{u}^{i}=L_{h}^{-\top} \underline{v}^{i}$ are the transformed eigenvectors and $u_{h}^{i} \in W_{h_{x}}$ are the related functions. With the inverse inequality

$$
\left\|\nabla_{x} v_{h}\right\|_{L^{2}(\Omega)}^{2} \leq c_{I} h_{x}^{-2}\left\|v_{h}\right\|_{L^{2}(\Omega)}^{2} \quad \text { for all } v_{h} \in W_{h_{x}},
$$


this condition is satisfied for

$$
c_{I} h_{x}^{-2}<12 h_{t}^{-2}
$$

In the particular case $d=1$, we have $c_{I}=12$, and therefore stability follows for

$$
h_{t}<h_{x} .
$$

When $W_{h_{x}} \subset H_{0}^{1}(\Omega)$ is also of tensor-product structure, for example when considering the spatial domain $\Omega=(0,1)^{d}$, we conclude $c_{I}=12 d$, and therefore the stability condition

$$
h_{t}<\frac{h_{x}}{\sqrt{d}} .
$$

As numerical example, we consider for $d=2$ the spatial domain $\Omega=(0,1)^{2}$ and the exact solution

$$
u\left(x_{1}, x_{2}, t\right)=t^{2} \sin \left(\pi x_{1}\right) \sin \left(\pi x_{2}\right) \quad \text { for }\left(x_{1}, x_{2}, t\right) \in Q=\Omega \times(0, T)
$$

with $T=\frac{1}{\sqrt{2}}$. Then, stability follows when choosing

$$
\frac{h_{t}}{h_{x}}<\frac{1}{\sqrt{2}} \approx 0.7071068
$$

and we observe optimal orders of convergence even for the limit case of the CFL condition (5.6); see Table 5.1. Note that numerical experiments indicate that the stability condition (5.6) is sharp; see [45].

TABLE 5.1

Numerical results for the Galerkin-Bubnov formulation (5.5) for $Q=(0,1)^{2} \times\left(0, \frac{1}{\sqrt{2}}\right)$ for the limit case of the CFL condition (5.6).

\begin{tabular}{rccccccr} 
dof & $h_{x}$ & $h_{t}$ & $\left\|u-u_{h}\right\|_{L^{2}}$ & eoc & $\left|u-u_{h}\right|_{H^{1}}$ & eoc & $\kappa_{2}$ \\
\hline 2 & 0.500000 & 0.3535534 & 0.020970 & - & 0.39813 & - & 2.6 \\
36 & 0.250000 & 0.1767767 & 0.004890 & 2.1 & 0.19798 & 1.0 & 32.7 \\
392 & 0.125000 & 0.0883883 & 0.001199 & 2.0 & 0.09859 & 1.0 & 250.9 \\
3600 & 0.062500 & 0.0441942 & 0.000298 & 2.0 & 0.04924 & 1.0 & 1543.4 \\
30752 & 0.031250 & 0.0220971 & 0.000074 & 2.0 & 0.02461 & 1.0 & 8921.9 \\
254016 & 0.015625 & 0.0110485 & 0.000018 & 2.0 & 0.01231 & 1.0 & 50750.8
\end{tabular}

As for the scalar case and following [47], we can formulate and analyse a stabilised version of the variational formulation (5.5), which is unconditionally stable and which preserves the optimal order of convergence; see [38].

6. Conclusions. In this paper, we have formulated and analysed new non-standard variational formulations for finite element discretisations of parabolic and hyperbolic initial boundary value problems, in particular, for the heat and wave equations. Based on this analysis, we can analyse related boundary integral equations and boundary element methods, where we recover known results in the case of the heat equation [12], but we expect to derive new results in the case of the wave equation. Moreover, using this unified framework, it will be possible to analyse the coupling of space-time finite and boundary element methods. While the main focus of this paper was on the stability analysis of space-time variational formulations, 
much more work is required on the design of computationally efficient methods. This covers the formulation and analysis of inf-sup stable local basis functions for arbitrary space-time finite elements, of efficient and reliable a posteriori error estimators and adaptive schemes, and the construction and analysis of preconditioned parallel iterative solution strategies including domain decomposition methods.

Acknowledgement: The authors would like to thank the anonymous referees for their valuable suggestions and remarks.

\section{REFERENCES}

[1] R. ANDREEV, Stability of sparse space-time finite element discretizations of linear parabolic evolution equations, IMA J. Numer. Anal., 33 (2013), pp. 242-260.

[2] D. N. ARnold And P. J. Noon, Coercivity of the single layer heat potential, J. Comput. Math., 7 (1989), pp. 100-104.

[3] W. BANGERTH, M. GeIGER, AND R. RANNACHER, Adaptive Galerkin finite element methods for the wave equation, Comput. Meth. Appl. Math., 10 (2010), pp. 3-48.

[4] M. CostABEL, Boundary integral operators for the heat equation, Integral Equations Operator Theory, 13 (1990), pp. 498-552.

[5] L. BALES AND I. LASIECKA, Continuous finite elements in space and time for the nonhomogeneous wave equation, Comput. Math. Appl., 27 (1994), pp. 91-102.

[6] D. BRAESS, Finite Elemente. Theorie, Schnelle Löser und Anwendungen in der Elastizitätstheorie, Springer, Berlin, 2013.

[7] G. C. Cohen, Higher-Order Numerical Methods for Transient Wave Equations, Springer, Berlin, 2002.

[8] G. C. Cohen And S. Pernet, Finite Element and Discontinuous Galerkin Methods for Transient Wave Equations, Springer, Dordrecht, 2017.

[9] L. DemKowicz AND J. Gopalakrishnan, Analysis of the DPG method for the Poisson equation, SIAM J. Numer. Anal., 49 (2011), pp. 1788-1809.

[10] D. Devaud, Petrov-Galerkin space-time hp-approximation of parabolic equations in $H^{1 / 2}$, IMA J. Numer. Anal., published online, 2019. https://doi.org/10.1093/imanum/drz036

[11] D. Devaud And C. Schwab, Space-time hp-approximation of parabolic equations, Calcolo, 55 (2018), Art. 35, 23 pages.

[12] S. Dohr, O. STEInBACH, AND K. NiINO, Space-time boundary element methods for the heat equation, in Space-Time Methods. Applications to Partial Differential Equations, U. Langer and O. Steinbach, eds., Radon Series on Computational and Applied Mathematics, vol. 25, de Gruyter, Berlin, 2019, pp. 1-60.

[13] W. DöRFLER, S. FINDEISEN, AND C. WIENERS, Space-time discontinuous Galerkin discretizations for linear first-order hyperbolic evolution systems Comput. Methods Appl. Math., 16 (2016), pp. 409-428.

[14] A. ERn AND J.-L. Guermond, Theory and Practice of Finite Elements, Springer, New York, 2004.

[15] A. ERn, I. SMEARs, AND M. VohraliK, Guaranteed, locally space-time efficient, and polynomial-degree robust a posteriori error estimates for high-order discretizations of parabolic problems, SIAM J. Numer. Anal., 55 (2017), pp. 2811-2834.

[16] M. Fontes, Parabolic Equations with Low Regularity, PhD. Thesis, Department of Mathematics, Lund Institute of Technology, Lund, 1996.

[17] D. A. FRENCH, AND T. E. PETERSON, A continuous space-time finite element method for the wave equation, Math. Comp., 65 (1996), pp. 491-506.

[18] E. Hairer, S. P. NøRSETt, AND G. WANNER, Solving Ordinary Differential Equations. I., 2nd ed., Springer, Berlin, 1993.

[19] D. HilberT, Ein Beitrag zur Theorie des Legendreschen Polynoms, Acta Math., 18 (1894), pp. 155-159.

[20] F. W. King, Hilbert Transforms. Vol. 1., Cambridge University Press, Cambridge, 2009.

[21] U. Köcher AND M. BAUSE, Variational space-time methods for the wave equation, J. Sci. Comput., 61 (2014), pp. 424-453.

[22] O. A. Ladyzhenskaya, The Boundary Value Problems of Mathematical Physics, Springer, New York, 1985.

[23] O. A. Ladyzhenskaya, V. A. Solonnikov, AND N. N. URAL'TSEVA, Linear and Quasilinear Equations of Parabolic Type, American Mathematical Society, Providence, 1968.

[24] U. LANGer, S. E. MOORE, AND M. NeUmÜLLeR, Space-time isogeometric analysis of parabolic evolution problems, Comput. Methods Appl. Mech. Engrg., 306 (2016), pp. 342-363.

[25] S. LARSSON AND C. SCHWAB, Compressive space-time Galerkin discretizations of parabolic partial differential equations, Preprint on arXiv, 2015. https://arxiv.org/abs/1501.04514

[26] U. LANGER AND M. WOLFMAYR, Multiharmonic finite element analysis of a time-periodic parabolic optimal control problem, J. Numer. Math., 21 (2013), pp. 265-300.

[27] J.-L. Lions And E. Magenes, Non-Homogeneous Boundary Value Problems and Applications. Vol. I., 


\section{ETNA}

Kent State University and

Johann Radon Institute (RICAM)

Springer, New York, 1972.

[28] - Non-Homogeneous Boundary Value Problems and Applications. Vol. II., Springer, New York, 1972.

[29] A. Moiola And I. Perugia, A space-time Trefftz discontinuous Galerkin method for the acoustic wave equation in first-order formulation, Numer. Math., 138 (2018), pp. 389-435.

[30] C. Mollet, Stability of Petrov-Galerkin discretizations: application to the space-time weak formulation for parabolic evolution problems, Comput. Meth. Appl. Math., 14 (2014), pp. 231-255.

[31] M. NeumÜller, Space-time methods: Fast solvers and applications, Monographic Series TU Graz/Computation in Engineering and Science vol. 20, Verlag der Technischen Universität Graz, Graz, 2013.

[32] A. PAZY, Semigroups of Linear Operators and Applications to Partial Differential Equations, Springer, New York, 1983.

[33] S. RJASANOW AND O. Steinbach, The Fast Solution of Boundary Integral Equations, Springer, New York, 2007.

[34] C. SCHWAB AND R. STEVENSON, Space-time adaptive wavelet methods for parabolic evolution problems, Math. Comp., 78 (2009), pp. 1293-1318.

[35] - Fractional space-time variational formulations of (Navier-) Stokes equations, SIAM J. Math. Anal., 49 (2017), pp. 2442-2467.

[36] O. SteInBACH, Numerical Approximation Methods for Elliptic Boundary Value Problems. Finite and Boundary Elements, Springer, New York, 2008.

[37] _ Space-time finite element methods for parabolic problems, Comput. Methods Appl. Math., 15 (2015), pp. 551-566.

[38] O. STEINBACH AND M. ZANK, A stabilized space-time finite element method for the wave equation, In: Advanced Finite Element Methods with Applications. Selected papers from the 30th Chemnitz FEM Symposium 2017, T. Apel, U. Langer, A. Meyer, and O. Steinbach, eds., Lecture Notes in Computational Science and Engineering, vol. 128, Springer, Cham, 2019, pp. 341-370.

[39] - A note on the efficient evaluation of a modified Hilbert transformation, Tech. Report, Berichte aus dem Institut für Angewandte Mathematik, Bericht 2019/8, TU Graz, Graz, 2019.

[40] _ Inf-sup stable variational formulations for the wave equation, in preparation.

[41] R. STEVENSON AND J. WESTERDIEP, Stability of Galerkin discretizations of a mixed space-time variational formulation of parabolic evolution equations, IMA J. Numer. Anal., drz069, 2020. https://doi.org/10.1093/imanum/drz069

[42] V. ThomÉE, Galerkin finite element methods for parabolic problems, 2nd ed., Springer, Berlin, 2006.

[43] K. URBAN AND A. T. PATERA, An improved error bound for reduced basis approximation of linear parabolic problems, Math. Comp., 83 (2014), pp. 1599-1615.

[44] J. WLOKA, Partielle Differentialgleichungen: Sobolevräume und Randwertaufgaben, Teubner, Stuttgart, 1982.

[45] M. ZANK, Inf-Sup Stable Space-Time Methods for Time-Dependent Partial Differential Equations, Monographic Series TU Graz/Computation in Engineering and Science, vol. 36, Verlag der Technischen Universität Graz, Graz, 2020.

[46] E. ZEIDLER, Nonlinear Functional Analysis and its Applications. II/A, Springer, New York, 1990.

[47] A. A. ZLOTNIK, Convergence rate estimates of finite-element methods for second-order hyperbolic equations, In: Numerical Methods and Applications, G. I. Marchuk, ed., CRC, Boca Raton, 1994, pp. 155-220. 